\title{
THE COUPLING OF MOTION AND CONDUCTIVE HEATING OF A GAS BY LOCALIZED ENERGY SOURCES*
}

\author{
ANTONIO L. SÁNCHEZ ${ }^{\dagger}$, JOSÉ L. JIMÉNEZ-ÁLVAREZ ${ }^{\dagger}$, AND AMABLE LIÑÁN
}

\begin{abstract}
This paper investigates the time evolution of the near-isobaric flow field produced in a gas after the sudden application of a constant heat flux from a localized energy source. The problems of plane, line, and point heat sources are all investigated, with a power law for the temperature dependence of the thermal conductivity, after reduction to a quasi-linear heat equation for the temperature. In the planar and spherical cases, the constant heat flux defines scales for the length and time, which are used to nondimensionalize the problem. Numerical integration is used to provide the evolution of the temperature and velocity, and limiting solutions corresponding to small and large rescaled times are obtained. In the axisymmetric case, due to the absence of characteristic length and time scales, the solution is seen to admit a self-similar description in terms of the nondimensional heat flux. Profiles of temperature and radial velocity are provided for different values of this parameter, and the asymptotic limits of both small and large heating rates are addressed separately. The analysis reveals, in particular, the existence of front solutions when the resulting temperatures become much larger than the initial temperature, as occurs for sufficiently large times for the planar source, for sufficiently small times for the point source, and for sufficiently large heating rates for the line source.
\end{abstract} tions

Key words. self-similar solutions, asymptotic methods, nonlinear heat conduction, front solu-

AMS subject classifications. 34E15, 80A32

PII. S0036139902403895

1. Introduction. The expansion accompanying the heating of a gas after the application of an energy source sets the fluid in motion away from the source. The purpose of this paper is to give a description of the associated nonlinear heating process when the induced velocities are much smaller than the velocity of sound, so that one can neglect pressure variations in the first approximation. Furthermore, the analysis treats the energy source as being of negligible size and neglects the effect of gravity, two simplifications that are simultaneously valid when the size of the heated region is much larger than that of the energy source and still sufficiently small so that the buoyancy-induced velocity remains smaller than the thermal-expansion velocity.

We shall consider the one-dimensional transient solutions appearing with plane, line, and point energy sources when a constant heat flux is applied. Numerical and asymptotic techniques will be employed to describe the evolution with time of the temperature and velocity fields. The solution will be seen to depend on the combined effect of outward convection, due to the gas expansion, and of nonlinear heat conduction, associated with the temperature dependence of the thermal conductivity. The results of the analysis should be useful for understanding the ignition process of a reactive gas mixture by a localized energy source, as can be realized in practice by a

\footnotetext{
* Received by the editors March 11, 2002; accepted for publication (in revised form) September 13, 2002; published electronically February 25, 2003. This research was supported by the Fifth Framework program of the European Commission under the Energy, Environment, and Sustainable Development contract EVG1-CT-2001-00042 EXPRO and by the Spanish MCYT under project 20014603-E.

http://www.siam.org/journals/siap/63-3/40389.html

†Departamento de Ingeniería Térmica y de Fluidos, Universidad Carlos III de Madrid, 28911 Leganés, Spain (asanchez@ing.uc3m.es).

${ }_{\ddagger}^{\ddagger}$ Departamento de Motopropulsión y Termofluidodinámica, E. T. S. I. Aeronáuticos, Universidad Politécnica de Madrid, 28040 Madrid, Spain (linan@tupi.dmt.upm.es).
} 
laser beam or by passing an electric current through a thin wire.

A simple order-of-magnitude analysis serves to anticipate the characteristic scales of the problem. Consider an energy source located in an infinite gas medium at rest with initial temperature and density $\mathrm{T}_{o}$ and $\rho_{o}$, respectively. If a constant energy flux is applied after time $t=0$, the characteristic time $t_{c}$ required to heat a region of characteristic size $\mathrm{r}_{c}$, so that the temperature is increased by an amount of order $\mathrm{T}_{o}$, is determined by the energy balance

$$
\mathrm{q}_{j} \mathrm{t}_{c} \sim \rho_{o} c_{p} \mathrm{~T}_{o} \mathrm{r}_{c}^{j+1}
$$

where $c_{p}$ is the specific heat at constant pressure, assumed to be constant, and the index $j$ takes the values $j=(0,1,2)$ for planar, cylindrical, and spherical geometries. Correspondingly, $\mathrm{q}_{0}, \mathrm{q}_{1}$, and $\mathrm{q}_{2}$ represent, respectively, the heating rate per unit surface for the planar source, the heating rate per unit length for the line source, and the heating rate of the point source. The above equation must be supplemented by the condition

$$
\mathrm{q}_{j} \sim \mathrm{r}_{c}^{j-1} k_{o} \mathrm{~T}_{o},
$$

which states that the energy flux is conducted across the heated region, with $k_{o}$ representing the value of the thermal conductivity at the initial temperature $T_{o}$.

For the planar and point sources, the above two balances give the characteristic scales of length and time

$$
\mathrm{r}_{c} \sim\left(\frac{\mathrm{q}_{j}}{k_{o} \mathrm{~T}_{o}}\right)^{1 /(j-1)} \text { and } \mathrm{t}_{c} \sim \alpha_{o}^{-1}\left(\frac{\mathrm{q}_{j}}{k_{o} \mathrm{~T}_{o}}\right)^{2 /(j-1)},
$$

where $\alpha_{o}=k_{o} /\left(\rho_{o} c_{p}\right)$ is the unperturbed thermal diffusivity. On the other hand, the characteristic velocity due to thermal expansion associated with relative changes in density of order unity, which can be anticipated from the continuity equation to be of order $\mathrm{v}_{c}=\mathrm{r}_{c} / \mathrm{t}_{c}$, becomes in this case

$$
\mathrm{v}_{c} \sim \alpha_{o}\left(\frac{\mathrm{q}_{j}}{k_{o} \mathrm{~T}_{o}}\right)^{-1 /(j-1)} .
$$

As shown below, use of these scales enables the problems $j=0$ and $j=2$ to be written in a convenient parameter-free form. On the other hand, no characteristic scales can be constructed for the line source, for which the radial extent of the heated region increases with time according to

$$
\mathrm{r}_{c} \sim\left[\frac{\mathrm{q}_{1}}{\left(k_{o} \mathrm{~T}_{o}\right)}\right]^{1 / 2}\left(\alpha_{o} \mathrm{t}\right)^{1 / 2},
$$

while the characteristic velocity is given by

$$
\mathrm{v}_{c} \sim\left[\frac{\mathrm{q}_{1}}{\left(k_{o} \mathrm{~T}_{o}\right)}\right]^{1 / 2}\left(\alpha_{o} / \mathrm{t}\right)^{1 / 2} .
$$

Because of the absence of characteristic scales, the problem will be seen to admit a similarity solution in terms of the self-similar coordinate $\mathrm{r} /\left(\alpha_{o} \mathrm{t}\right)^{1 / 2}$, with $q=\mathrm{q}_{1} /\left(2 \pi k_{o} \mathrm{~T}_{o}\right)$ entering as a governing parameter. 
The ranges of validity for the different assumptions employed in the paper can be delineated by using the above scaling laws (1.3)-(1.6). For instance, the assumption that the source is localized is valid only when $r_{c}$ is much larger than the size of the energy source, while gravity-induced velocities, of order $\left(g \mathrm{r}_{c}\right)^{1 / 2}$, can be neglected only when $\mathrm{v}_{c} \gg\left(g \mathrm{r}_{c}\right)^{1 / 2}$ for $\mathrm{r}_{c} \ll\left(\alpha_{o}^{2} / g\right)^{1 / 3}$. The assumption of isobaric heating holds only when the induced velocities $v_{c}$ are much smaller than the velocity of sound, given in order of magnitude by $\left(c_{p} \mathrm{~T}_{o}\right)^{1 / 2}$, thereby producing pressure variations, of order $\rho_{o} v_{c}^{2}$, that are much smaller than the ambient value. It should also be noted that the above considerations provide, for a given gas mixture, the range of heating rates for which the analyses of the planar and point sources remain accurate. On the other hand, since the characteristic scales given in (1.5)-(1.6) change with time, the above considerations give the time range for which the analysis of the line source holds.

Neither finite-size sources nor buoyancy and compressibility effects are addressed in the present work. When gravity enters, the symmetric solution determined here is expected to evolve to give a steady plume for large times, giving a flow pattern that has been extensively studied in the past (see, e.g., $[8,9]$ for entries into the literature of thermal plumes from line and point sources). When compressibility effects are significant, a strong shock wave can be expected to form, a phenomenon also observed following the instantaneous localized deposition of a finite amount of energy $[18,19,20]$. This shock wave weakens as it moves away from the source, eventually leading to an acoustic wave as the pressure settles everywhere to the ambient value for sufficiently large times.

The structure of the paper is as follows. After formulating the problem, we will address the similarity solution emerging in the case of a line source. The selfsimilar temperature and velocity profiles will be given for different values of the heat release rate, and the asymptotic limit of large heat release rates will be described in detail. Next, we will present the solution corresponding to planar and point sources, which involve integration of a parameter-free nonlinear parabolic equation for the temperature. The analysis is extended to include the asymptotic limits of small and large rescaled times. Finally, some concluding remarks will be given.

It should be noted that the problem of near-isobaric heat propagation in a gas from a plane source was addressed previously by Clarke, Kassoy, and Riley in their study of heating of a gas slot confined between infinite parallel walls [5]. In particular, the nonlinear heat equation that governs the problem was derived. They showed that, when the heating rate is applied for a sufficiently long time, the characteristic temperature of the heated region becomes much larger than $\mathrm{T}_{o}$, so that the thermal wave becomes a front solution with an edge that clearly defines the hot region surrounding the source. We shall see that a front solution also appears with the line source in the limit of large heat release rates and with the point source for sufficiently small times. As seen below, the structure of the solution includes a locally planar thin layer of warm gas, identical for all three configurations, that separates the region of hot gas from the outer cold gas, at temperature $\mathrm{T}=\mathrm{T}_{o}$. It is worth mentioning that similar front solutions have been previously identified in asymptotic analyses of heat conduction problems when the thermal conductivity depends strongly on the temperature [22], e.g., in electronic conduction in plasmas [16, 23], or in the presence of large temperature variations in gases, as occurs in supercritical droplet evaporation [15]. Front solutions are also encountered in problems of mass diffusion [7] and in flows in porous media [2]. 
2. Formulation. In the near-isobaric limit considered here, the momentum equation becomes secondary for the computation of the one-dimensional problems addressed, in the sense that the resulting velocity and temperature fields can be determined by integrating the continuity equation

$$
\frac{\partial \rho}{\partial \mathrm{t}}+\frac{1}{\mathrm{r}^{j}} \frac{\partial}{\partial \mathrm{r}}\left(\mathrm{r}^{j} \rho \mathrm{v}\right)=0
$$

and the energy equation

$$
\frac{\partial}{\partial \mathrm{t}}\left(\rho c_{v} \mathrm{~T}\right)+\frac{1}{\mathrm{r}^{j}} \frac{\partial}{\partial \mathrm{r}}\left(\mathrm{r}^{j} \rho \mathrm{v} c_{p} \mathrm{~T}-\mathrm{r}^{j} k \frac{\partial \mathrm{T}}{\partial \mathrm{r}}\right)=0,
$$

supplemented with the equation of state for the ideal gas

$$
\rho \mathrm{T}=\rho_{o} \mathrm{~T}_{o},
$$

which is written with pressure differences neglected. These pressure differences, which are much smaller than the ambient pressure in this near-isobaric limit, can be computed a posteriori by integrating the momentum balance equation. In the formulation, $\rho, \mathrm{T}$, and $\mathrm{v}$ denote, respectively, the density, temperature, and velocity of the gas, while $c_{v}$ represents the specific heat at constant volume. For generality, the thermal conductivity $k$ is allowed to vary in our analysis from its initial value $k_{o}$, with a temperature dependence given by

$$
\frac{k}{k_{o}}=\left(\frac{\mathrm{T}}{\mathrm{T}_{o}}\right)^{\sigma}
$$

where the exponent $\sigma$ is typically in the range $0 \leq \sigma \leq 1$ in gases and takes the value $\sigma=5 / 2$ for electronic conduction in plasmas. The initial and boundary conditions for (2.1) and (2.2) corresponding to an infinitesimally small heat source located at $\mathrm{r}=0$ are

$$
\mathrm{t}=0, \quad \mathrm{r}>0: \mathrm{T}=\mathrm{T}_{o}, \quad \rho=\rho_{o}
$$

and

$$
\mathrm{t}>0\left\{\begin{aligned}
\mathrm{r}=0: & \mathrm{v}=0,-2^{j} \pi^{\delta_{j}} \mathrm{r}^{j} k \partial \mathrm{T} / \partial \mathrm{r}=\mathrm{q}_{j}, \\
\mathrm{r}=\infty: & \mathrm{T}=\mathrm{T}_{o},
\end{aligned}\right.
$$

where $\delta_{j}=0$ if $j=0$ and $\delta_{j}=1$ otherwise.

The approximation (2.3) eliminates the time derivative in (2.2), because in this limit of near-isobaric heating, the internal energy does not accumulate locally in the flow field. Integrating the resulting equation, using the boundary condition at $r=0$, yields

$$
2^{j} \pi^{\delta_{j}} \mathrm{r}^{j}\left(\mathrm{v} \rho_{o} c_{p} \mathrm{~T}_{o}-k \frac{\partial \mathrm{T}}{\partial \mathrm{r}}\right)=\mathrm{q}_{j}
$$

As can be seen, the heat released at $\mathrm{r}=0$ is transported partly by convection and partly by heat conduction. Introducing the dimensionless temperature $T=\mathrm{T} / \mathrm{T}_{o}=$ $\rho_{o} / \rho$ and substituting (2.7) into (2.1) finally gives

$$
\frac{1}{T^{2}} \frac{\partial T}{\partial \mathrm{t}}-\frac{1}{\mathrm{r}^{j}} \frac{\partial}{\partial \mathrm{r}}\left[\frac{\alpha_{o}}{T}\left(\frac{\mathrm{q}_{j}}{2^{j} \pi^{\delta_{j}} k_{o} \mathrm{~T}_{o}}+\mathrm{r}^{j} T^{\sigma} \frac{\partial T}{\partial \mathrm{r}}\right)\right]=0
$$


to be integrated with initial and boundary conditions

$$
\left\{\begin{array}{l}
\mathrm{t}=0: T=1 \\
t>0:\left\{\begin{array}{l}
\mathrm{r}=0: \mathrm{r}^{j} T^{\sigma} \partial T / \partial \mathrm{r}=-\mathrm{q}_{j} /\left(2^{j} \pi^{\delta_{j}} k_{o} \mathrm{~T}_{o}\right), \\
\mathrm{r}=\infty: T=1 .
\end{array}\right.
\end{array}\right.
$$

This nonlinear heat problem describes the buoyancy-free isobaric evolution of the gas temperature, subject to a localized energy source of rate $\mathrm{q}_{j}$, which can vary with time.

3. The line source of heat. As previously mentioned, for the line source the solution to $(2.8)$ when the heating rate $\mathrm{q}_{1}$ is constant is of the self-similar form $T=$ $T(\eta)$, involving the similarity variable $\eta=\mathrm{r} /\left(\alpha_{o} \mathrm{t}\right)^{1 / 2}$, so that $T(\eta)$ is given by the solution of

$$
\left[\eta^{-1}\left(q+\eta T^{\sigma} T_{\eta}\right)-\frac{\eta}{2}\right] T_{\eta}=T \eta^{-1}\left(\eta T^{\sigma} T_{\eta}\right)_{\eta} \begin{cases}\eta=0: & q+\eta T^{\sigma} T_{\eta}=0 \\ \eta=\infty: & T=1\end{cases}
$$

To simplify the notation throughout the text, subscripts will be utilized to denote differentiation with respect to a given variable, so that, for instance, $T_{\eta}=d T / d \eta$ in the above equation. Apart from the thermal-conductivity exponent $\sigma$, only the dimensionless heating rate

$$
q=\frac{\mathrm{q}_{1}}{2 \pi k_{o} T_{o}}
$$

enters as a parameter in (3.1). As can be seen, besides the thermal-expansion velocity

$$
u=\frac{\mathrm{v}}{\left(\alpha_{o} / \mathrm{t}\right)^{1 / 2}}=\frac{1}{\eta}\left(q+\eta T^{\sigma} T_{\eta}\right),
$$

the convective term incorporates an apparent negative velocity $-\eta / 2$ due to the growing length scale used in the definition of $\eta$.

Sample distributions of $T(\eta)$ are shown in Figure 1 for different values of $q$, with a value $\sigma=0.5$ adopted in the calculations for the temperature dependence of the conductivity. Integration by a shooting method was initiated near $\eta=0$, where the temperature profile is of the form

$$
T^{\sigma+1} \simeq-(\sigma+1) q \ln \eta+B,
$$

with $B(q, \sigma)$ representing an unknown constant that was varied in the shooting procedure to satisfy the boundary condition $T=1$ at $\eta=\infty$. The resulting value of $B$ is shown as an inset in Figure 1 for $\sigma=(0,0.5,1.0)$. Note that the local high-temperature description (3.4) can be of interest for the analysis of some related problems, such as the ignition of a reactive gas mixture by hot wires or by laser beams [10].

The temperature distribution can be used to determine from (3.3) the gas velocity induced by thermal expansion. This velocity is zero at the heat source and also at $\eta=\infty$ and reaches a maximum at an intermediate location, a result clearly seen in the velocity profiles exhibited in Figure 2. The effect of the heat source on the far field is that of a volumetric source of fluid, inducing radial velocities that decay according to $u \simeq q / \eta$ for $\eta \gg 1$.

The solution corresponding to small heating rates can be determined by introducing an expansion for $T-1$ in increasing powers of $q$. Since in this case both $\rho$ and $k$ change by only a small amount from their unperturbed values $\rho_{o}$ and $k_{o}$, the resulting solution is, in the first approximation, that corresponding to a solid with constant 


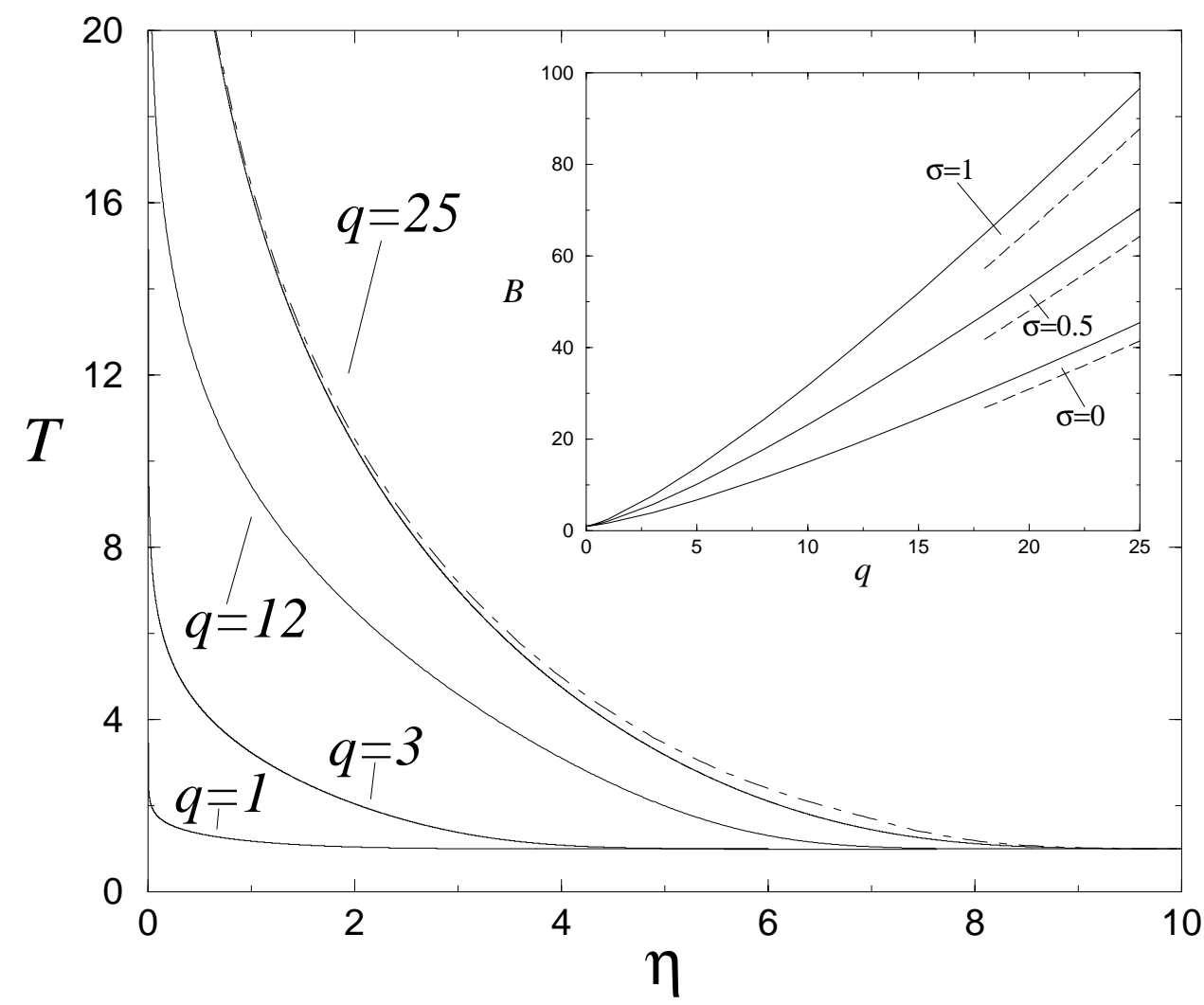

FIG. 1. The temperature profiles obtained by integration of (3.1) with $\sigma=0.5$ (solid lines) and from the large-q composite expansion (dot-dashed lines); the inset shows the variation with $q$ of the constant $B$, along with the large-q prediction $B=q[b+(\sigma+1) \ln (q) / 2]$.

thermal conductivity $(T-1) / q=\frac{1}{2} E_{1}\left(\eta^{2} / 4\right)$ (see [4]), where $E_{1}$ is the exponential integral function [1]. The effect of the thermal expansion emerges in the solution, giving a small modification of order $q^{2}$ to the temperature increment $T-1$ and inducing small radial velocities, of order $q$, that can be determined from (3.3) to give $u=(q / \eta)\left[1-\exp \left(-\eta^{2} / 4\right)\right]$. This description can be expected to fail as $T-1$ increases to values of order unity for $\eta \rightarrow 0$, in an exponentially small region around the axis corresponding to $\eta \sim \exp (-1 / q)$. This region can be studied by employing $\ln (\eta) / q$ as an appropriately stretched coordinate, an analysis that gives $T^{\sigma+1}=1-(\sigma+1) q \ln \eta$ as the leading-order representation for the temperature. This is in agreement with the results shown in the inset of Figure 1, where the constant $B$ approaches unity as $q \rightarrow 0$.

The analysis of the limit of large heat release rates, $q \gg 1$, is more complicated and requires consideration of separate spatial regions. As seen in Figure 1, both the extent of the heated domain and the characteristic value of the temperature grow with increasing values of $q$. A simple order-of-magnitude analysis of (3.1) reveals that the rescaled variables $\xi=q^{-1 / 2} \eta$ and $\theta=q^{-1 /(\sigma+1)} T$ are appropriate replacements for $\eta$ and $T$ in this limit of large $q$. Use of these alternative variables enables (3.1) to 


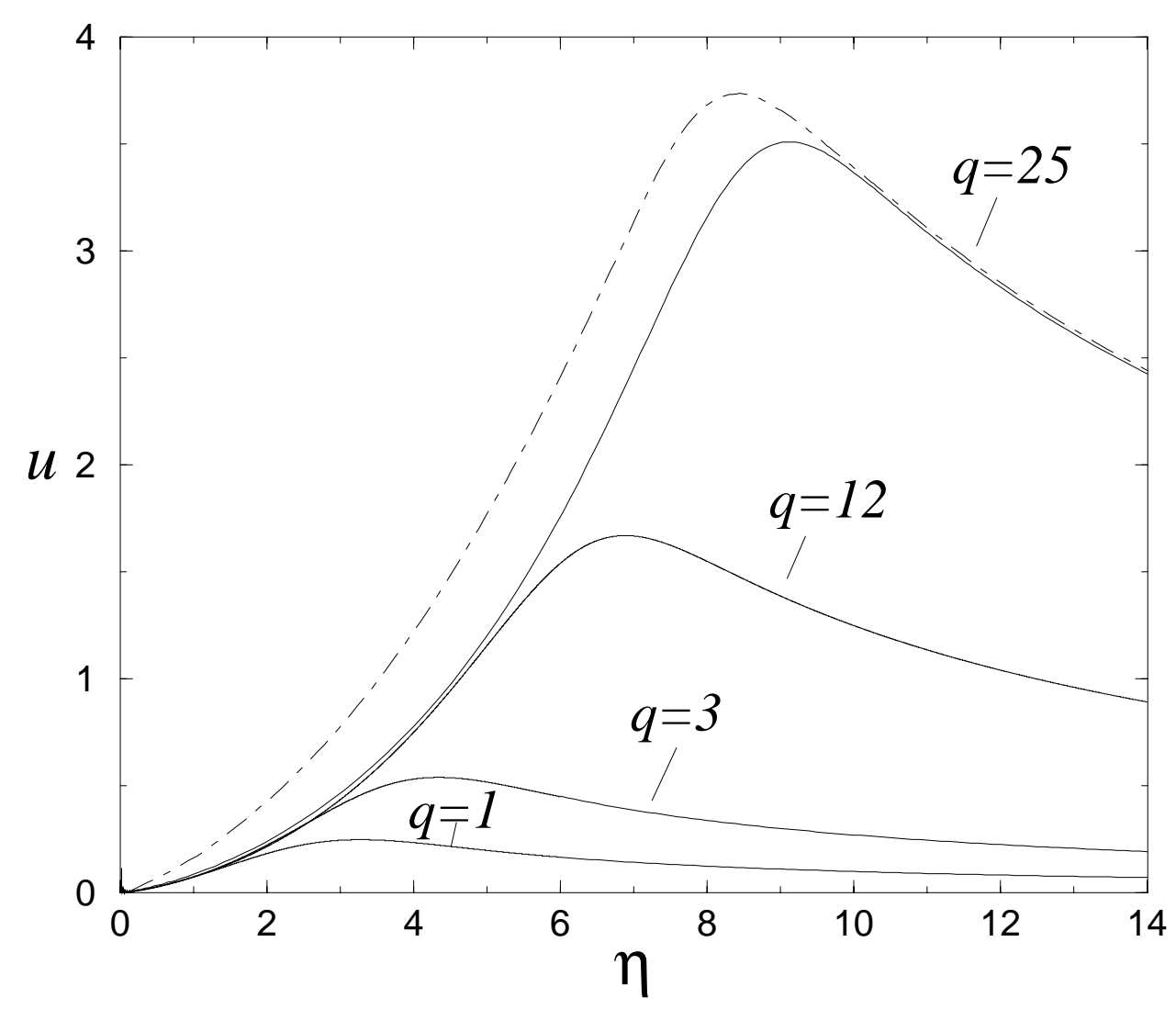

FIG. 2. The velocity distribution (3.3) for $\sigma=0.5$ evaluated with the temperature profiles determined numerically (solid lines) and with the large-q composite expansion (dot-dashed lines).

be written in the form

$$
\left[\xi^{-1}\left(1+\xi \theta^{\sigma} \theta_{\xi}\right)-\frac{\xi}{2}\right] \theta_{\xi}=\theta \xi^{-1}\left(\xi \theta^{\sigma} \theta_{\xi}\right)_{\xi}\left\{\begin{array}{l}
\xi=0: \xi \theta^{\sigma} \theta_{\xi}=-1 \\
\xi=\infty: \theta=q^{-1 /(\sigma+1)}
\end{array}\right.
$$

3.1. The high-temperature region. Introducing an expansion of the form

$$
\theta=\theta_{0}+q^{-\mu_{1}} \theta_{1}+\cdots
$$

into (3.5) produces a series of problems that can be solved sequentially, with the order $\mu_{1}$ for the first-order correction to the leading-order result being determined in the course of the analysis.

The problem emerging at leading order for the function $\theta_{0}$,

$$
\left[\xi^{-1}\left(1+\xi \theta_{0}^{\sigma} \theta_{0 \xi}\right)-\frac{\xi}{2}\right] \theta_{0 \xi}=\theta_{0} \xi^{-1}\left(\xi \theta_{0}^{\sigma} \theta_{0 \xi}\right)_{\xi}\left\{\begin{array}{l}
\xi=0: \xi \theta_{0}^{\sigma} \theta_{0 \xi}=-1 \\
\xi=\sqrt{2}: \theta_{0}=0
\end{array}\right.
$$

has a front solution that neatly defines the hot region. The location $\xi=\sqrt{2}$ of the front is determined a priori from inspection of (3.7) by noting that heat conduction vanishes as the temperature approaches its zero boundary value, so that convection remains as the only transport mechanism there. Therefore, the leading edge of the 
temperature distribution must lie at $\xi=\sqrt{2}$, where the positive thermal-expansion velocity $U=q^{-1 / 2} u=\xi^{-1}\left(1+\xi \theta^{\sigma} \theta_{\xi}\right) \simeq \xi^{-1}$ equals the negative apparent velocity $-\xi / 2$ associated with the growing length scale. The front nature of the solution is clearly a result of the vanishing boundary temperature seen with the scales of this hightemperature region, as occurs in other problems of high-temperature hydrodynamics $[13,15,22,23]$.

The resulting function $\theta_{0}$ is shown in Figure 3 for four different values of the thermal-conductivity exponent $\sigma=(0,0.5,1.0,2.5)$. The numerical integration was started at $\xi \ll 1$, where $\theta_{0}^{\sigma+1} \simeq-(\sigma+1) \ln \xi+b$, with $b=(0.0477,0.1557,0.2914,0.7637)$ for $\sigma=(0,0.5,1.0,2.5)$. Note that, in terms of this shooting parameter, the constant $B$ appearing in (3.4) can be expressed in the form $B=q[b+(\sigma+1) \ln (q) / 2]$, an asymptotic prediction tested in the inset of Figure 1 . The temperature profiles are seen to approach the cold boundary $\xi=\sqrt{2}$ according to

$$
\theta_{0}=\left(\frac{1+\sigma}{1-\sigma}\right)^{1 /(1+\sigma)}(\sqrt{2}-\xi)^{2 /(1+\sigma)}
$$

if $\sigma<1$, according to

$$
\theta_{0}=\sqrt{2}(\sqrt{2}-\xi)\left[\ln \left(\frac{1}{\sqrt{2}-\xi}\right)\right]^{1 / 2}
$$

if $\sigma=1$, and according to

$$
\theta_{0}=E(\sqrt{2}-\xi)^{1 / \sigma}
$$

if $\sigma>1$, where $E$ is a constant to be determined as part of the numerical integration (e.g., $E=2.7449$ for $\sigma=5 / 2$ ).

As previously mentioned, the order $\mu_{1}$ of the first-order correction to the leadingorder results must be determined as part of the solution. Although the boundary condition at $\xi=\infty$ given in (3.5) suggests $\mu_{1}=1 /(\sigma+1)$, corresponding to a correction in temperature $T$ of order unity, it is shown below that the necessary correction is in fact larger when $\sigma<1$. The cases $\sigma=1$ and $\sigma>1$, which give $\mu_{1}=1 /(\sigma+1)$, are treated separately in the appendixes.

The function $\theta_{1}$ satisfies the equation

$$
\left(1-\frac{\xi^{2}}{2}\right) \theta_{1 \xi}=2 \theta_{0}\left(\xi \theta_{0}^{\sigma-1} \theta_{0 \xi}\right)_{\xi} \theta_{1}+\theta_{0}^{2}\left(\xi \theta_{0}^{\sigma-1} \theta_{1 \xi}\right)_{\xi}+\theta_{0}^{2}\left(\xi(\sigma-1) \theta_{0}^{\sigma-2} \theta_{0 \xi} \theta_{1}\right)_{\xi}
$$

obtained from linearizing (3.5) about $\theta_{0}$. The corresponding boundary conditions are

$$
\xi=0: \quad \xi \theta_{0}^{1+\sigma} \theta_{1 \xi}-\sigma \theta_{1}=0
$$

and

$$
\xi=\sqrt{2}: \quad \theta_{1}=0 .
$$

In addition to the trivial solution $\theta_{1}=0$, for each value of $\mu_{1}$ the above problem admits a single nontrivial solution that can be determined aside from an arbitrary multiplicative factor. To discriminate the value of $\mu_{1}$, one needs to investigate the corner layer that appears at distances of order $q^{-1 / 2}$ about $\xi=\sqrt{2}$, where the temperature becomes of order unity. 


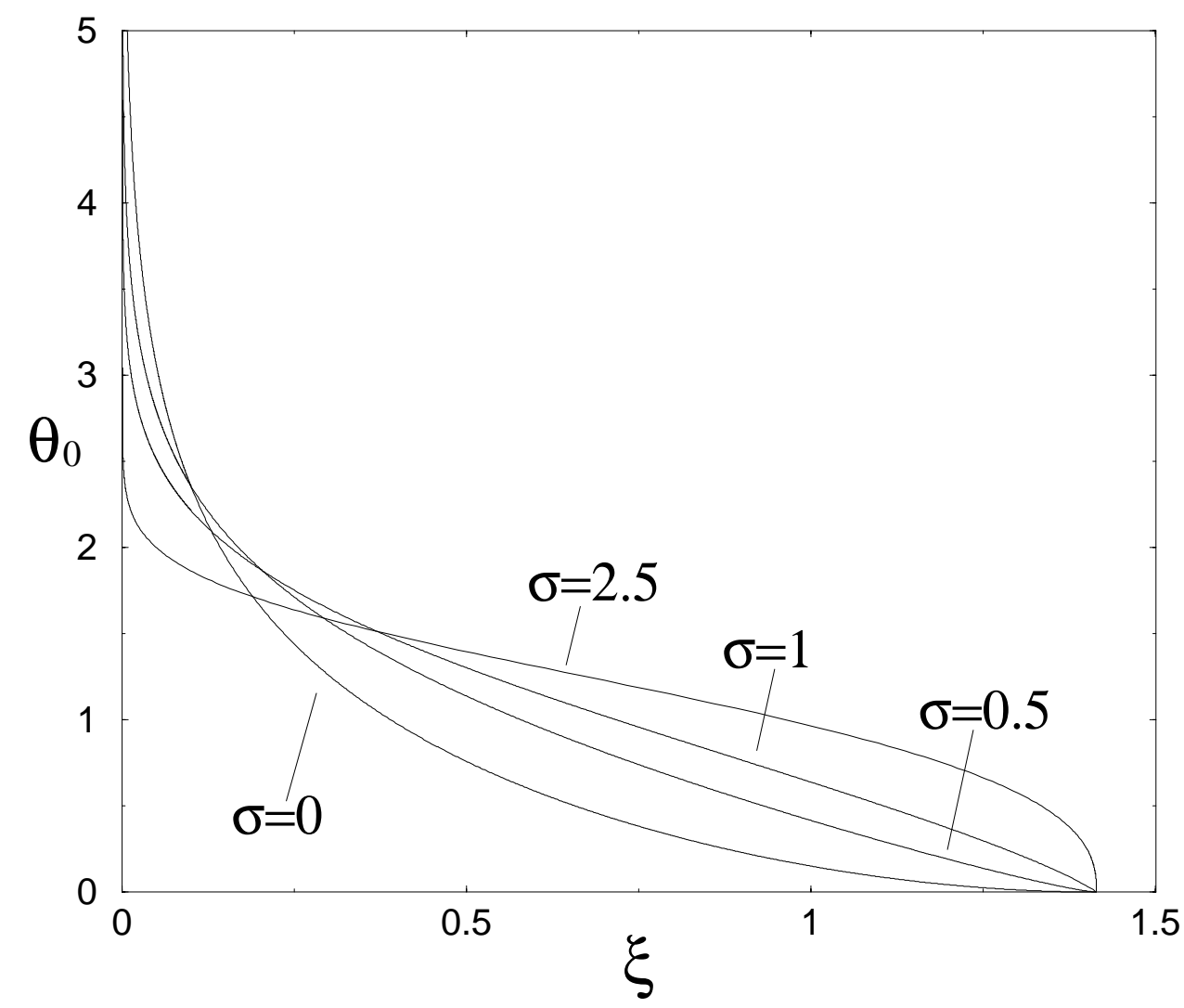

FIG. 3. The temperature profiles obtained by integration of (3.7) for $\sigma=(0,0.5,1.0,2.5)$.

3.2. The corner layer. Around $\xi=\sqrt{2}$ the temperature must evolve from the cold boundary distribution given in (3.8) to the final asymptotic value $T=1$. The description of the resulting corner layer must make use of the translated coordinate $\chi=\sqrt{2 q}-\eta$. At leading order the problem becomes

$$
T^{2}\left(T^{\sigma-1} T_{\chi}\right)_{\chi}+\chi T_{\chi}=0\left\{\begin{array}{l}
\chi \rightarrow-\infty: T=1, \\
\chi \rightarrow \infty: T \rightarrow\left(\frac{1+\sigma}{1-\sigma}\right)^{1 /(1+\sigma)} \chi^{2 /(1+\sigma)} .
\end{array}\right.
$$

The solution to this problem, which is given in Figure 4, determines in particular the asymptotic behavior for $\chi \rightarrow \infty$, where the temperature is seen to approach only slowly its boundary value according to

$$
T-\left(\frac{1+\sigma}{1-\sigma}\right)^{1 /(1+\sigma)} \chi^{2 /(1+\sigma)}=A \chi^{\left(2-\sigma-\sqrt{2-\sigma^{2}}\right) /(1+\sigma)},
$$

with $A$ being a constant determined as part of the integration. Sample values are $A=(3.816,4.5080,5.664,9.353)$ for $\sigma=(0,0.25,0.5,0.75)$.

3.3. Uniformly valid description. Matching the solution given in (3.15) with the outer expansion $\theta=\theta_{0}+t^{-\mu_{1}} \theta_{1}+\cdots$ gives

$$
\mu_{1}=\frac{\sigma+\sqrt{2-\sigma^{2}}}{2(1+\sigma)}
$$




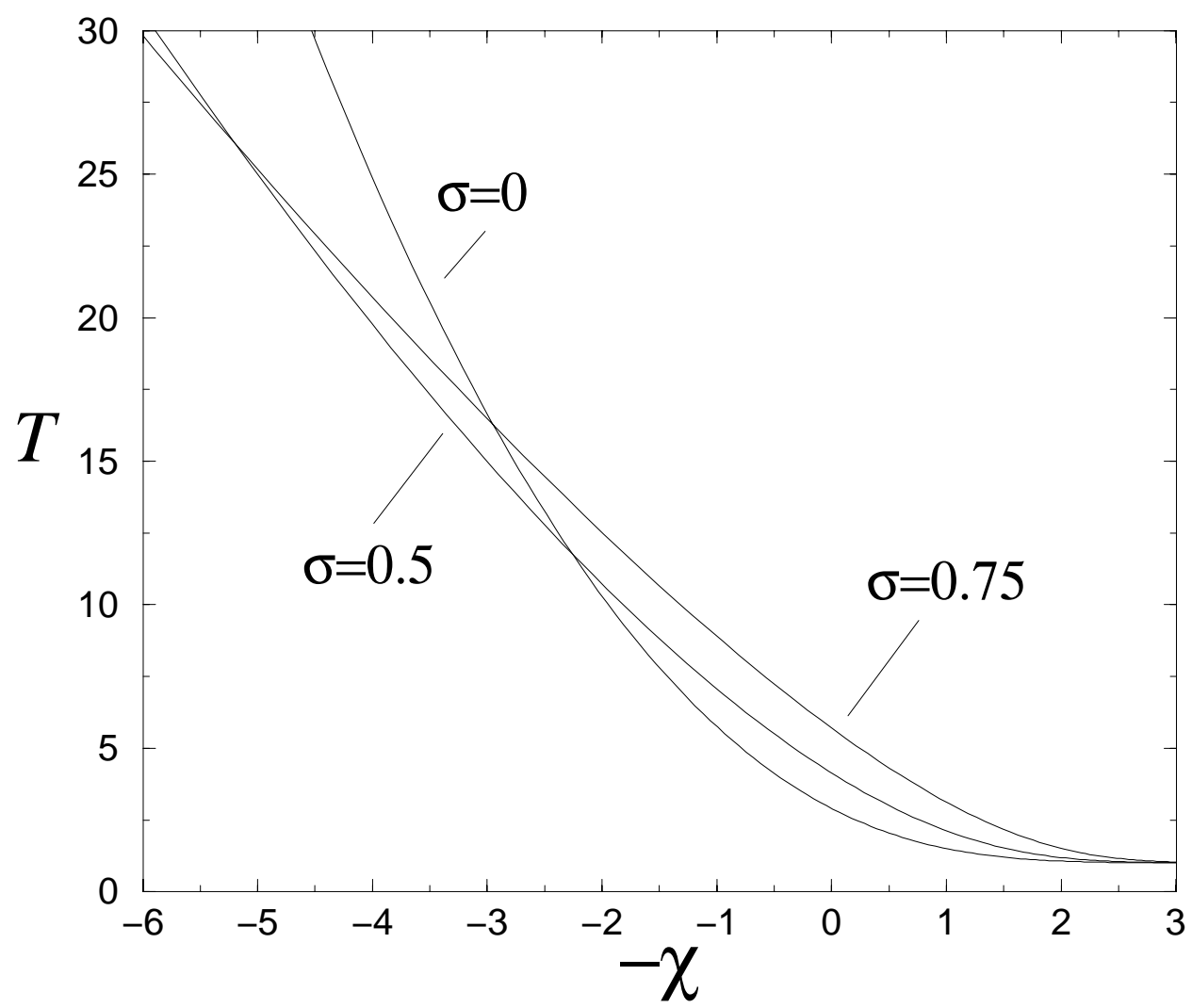

FIG. 4. The temperature profile across the transition layer for different values of $\sigma$.

for the order of the correction. Furthermore, the matching procedure provides

$$
\xi \rightarrow \sqrt{2}: \quad \theta_{1}=A(\sqrt{2}-\xi)^{\left(2-\sigma-\sqrt{2-\sigma^{2}}\right) /(1+\sigma)}
$$

as a replacement for (3.13), thereby removing all previously noted arbitrariness; i.e., (3.11) subject to (3.12) and (3.17) has a unique solution that must be determined by numerical integration.

A uniformly valid description for the temperature field can be obtained by combining the outer expansion for $\theta$ with the corner-layer profile according to the composite expansion

$$
T(\eta)=q^{1 /(\sigma+1)}\left[\theta_{0}(\xi)+q^{-\mu_{1}} \theta_{1}(\xi)\right]
$$

$$
+T(\chi)-H(\sqrt{2}-\xi)\left[\left(\frac{1+\sigma}{1-\sigma}\right)^{1 /(1+\sigma)} \chi^{2 /(1+\sigma)}-A \chi^{\left(2-\sigma-\sqrt{2-\sigma^{2}}\right) /(1+\sigma)}\right]
$$

where $H(\sqrt{2}-\xi)$ is the Heaviside function with origin $\xi=\sqrt{2}$ and $T(\chi)$ is the temperature profile across the corner layer, with the rescaled variables $\xi=q^{-1 / 2} \eta$ and $\chi=\sqrt{2 q}-\eta$ being those utilized above for the outer region and for the corner layer, respectively. The resulting temperature profile and the accompanying velocity 
profile, determined by straightforward substitution of (3.18) into (3.3), are plotted in Figures 1 and 2, showing reasonable agreement for the relatively large value of $q=25$ considered.

4. Constant heat flux from a plane wall. Use of the characteristic scales identified above in (1.3) and (1.4) provides $t=\alpha_{o}\left[\mathrm{q}_{0} /\left(k_{o} T_{o}\right)\right]^{2} \mathrm{t}, r=\left[\mathrm{q}_{0} /\left(k_{o} T_{o}\right)\right] \mathrm{r}$, and $v=\mathrm{v} /\left[\mathrm{q}_{0} \alpha_{o} /\left(k_{o} T_{o}\right)\right]$ as dimensionless variables to describe the planar source. As first shown by Clarke, Kassoy, and Riley [5], the problem then reduces to that of integrating

$$
\frac{\partial T}{\partial t}-T^{2} \frac{\partial}{\partial r}\left[\frac{1}{T}\left(1+T^{\sigma} \frac{\partial T}{\partial r}\right)\right]=0
$$

with initial condition

$$
t=0, \quad 0 \leq r<\infty: T=1
$$

and boundary conditions

$$
t>0 \begin{cases}r=0: & T^{\sigma} \partial T / \partial r=-1 \\ r=\infty: & T=1\end{cases}
$$

while the velocity can be computed from (2.7) to give

$$
v=1+T^{\sigma} \frac{\partial T}{\partial r}
$$

4.1. Temperature and velocity distributions. As can be seen, $\sigma$ is the only parameter left in the problem. An exact solution is known only for $\sigma=1$ (see [5]), a case for which the density-weighted coordinate $\mathrm{d} z=T^{-1} \mathrm{~d} r$, often introduced for the analysis of variable-density boundary layers [17, 21], reduces $(4.1)-(4.3)$ to the constant-density problem (see [4]), thereby yielding

$$
T=1+2 t^{1 / 2} \mathrm{i}^{1} \operatorname{erfc}\left(\frac{z}{2 t^{1 / 2}}\right) \text { and } r=z+t\left[1-4 \mathrm{i}^{2} \operatorname{erfc}\left(\frac{z}{2 t^{1 / 2}}\right)\right]
$$

as an implicit representation for the temperature profile $T(r)$, where $\mathrm{i}^{1} \mathrm{erfc}$ and $\mathrm{i}^{2} \mathrm{erfc}$ denote repeated integrals of the complementary error function erfc (see [1]). Correspondingly, the velocity profile (4.4) reduces to $v=1-\operatorname{erfc}\left[\int_{0}^{r} T^{-1} \mathrm{~d} r /\left(2 t^{1 / 2}\right)\right]$.

For $\sigma \neq 1$, the problem needs numerical integration. To handle the unbounded value of $\partial T / \partial t$ at $t=0$, the initial condition (4.2) must be replaced in the numerical integrations with the leading-order representation of the temperature profile for $t \ll 1$, when the temperature increase from the initial value $T=1$ is small, of order $t^{1 / 2}$, and is seen to be confined to a thin layer of characteristic thickness $t^{1 / 2}$ located in the vicinity of the wall. To describe this initial period it is convenient to introduce the self-similar variables $r / t^{1 / 2}$ and $(T-1) / t^{1 / 2}$ into (4.1)-(4.3), yielding in the first approximation the constant-density result (see [4]),

$$
\frac{(T-1)}{t^{1 / 2}}=2 \mathrm{i}^{1} \operatorname{erfc}\left[\frac{r}{\left(2 t^{1 / 2}\right)}\right]
$$

while the initial velocity distribution becomes

$$
v=1-\operatorname{erfc}\left(\frac{r}{2 t^{1 / 2}}\right)
$$

Characteristic temperature profiles obtained for $\sigma=0.5$ by numerical integration of (4.1) with boundary conditions (4.3) and with the initial profile (4.6) evaluated at $t \ll$ 1 are shown in Figure 5, along with the accompanying velocity profiles determined by evaluating (4.4). The profiles at $t=0.4$ are compared with the asymptotic predictions 


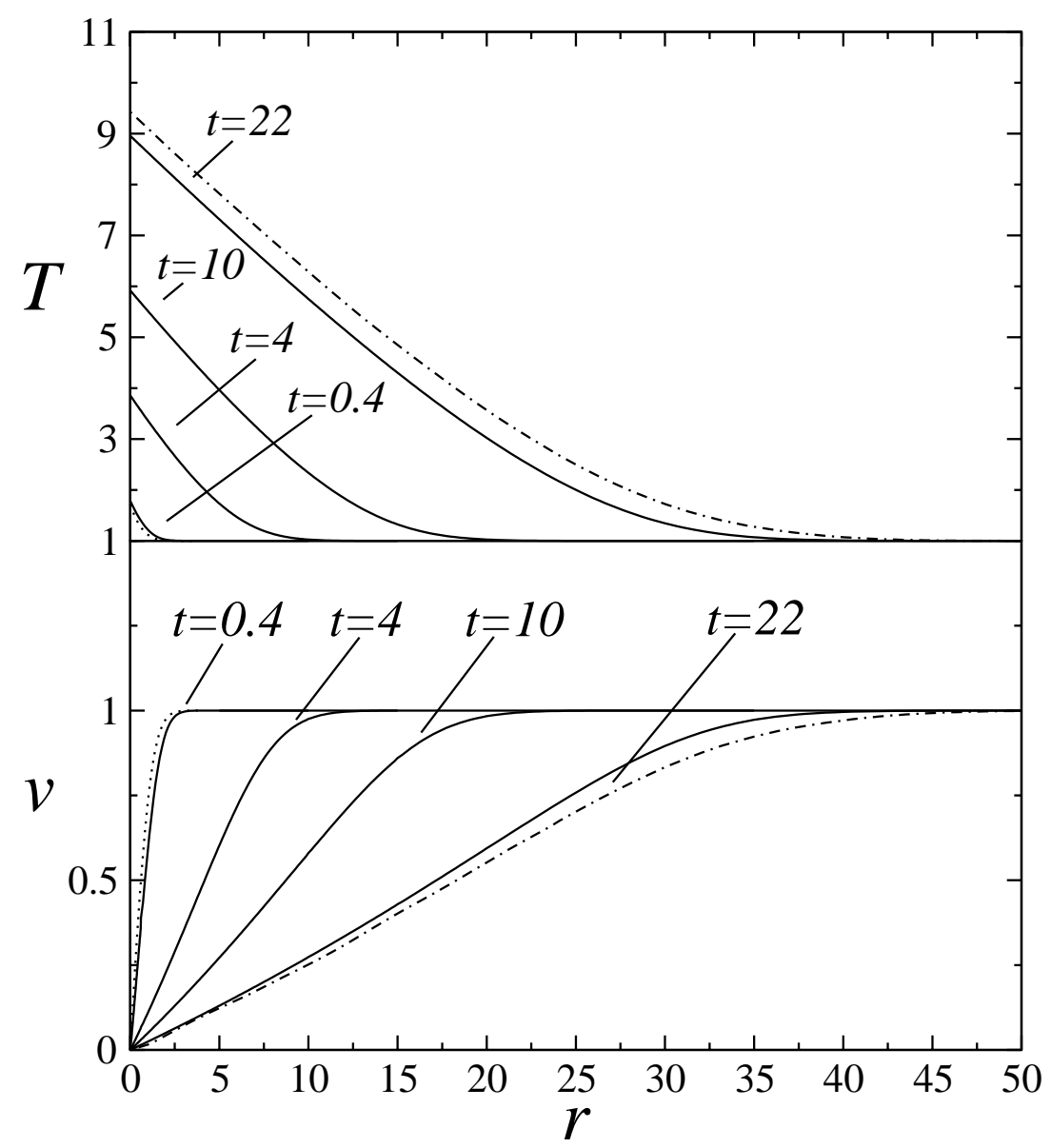

FIG. 5. The temperature and velocity profiles obtained by integration of (4.1)-(4.3) for $\sigma=0.5$ (solid lines); the profiles at $t=0.4$ are compared with the asymptotic predictions given in (4.6) and (4.7) for $t \ll 1$, and the profiles at $t=22$ are compared with the asymptotic predictions for $t \gg 1$.

for small times given in (4.6) and (4.7), while the profile $t=22$ is compared with the asymptotic prediction for $t \gg 1$, to be developed below.

In this planar case, the temperature remains bounded everywhere, growing with time. The evolution of the maximum temperature $T_{w}$ attained at the wall is shown in Figure 6 for $\sigma=0$ and $\sigma=0.5$. The numerical solution is compared with the asymptotic description for small $t$,

$$
T_{w}=1+\frac{2}{\pi^{1 / 2}} t^{1 / 2},
$$

obtained by evaluating (4.6) at $r=0$, and also with the results given below for $t \gg 1$. Note that (4.8) gives exactly the wall temperature at all times when $\sigma=1$, as can be seen by evaluating (4.5) at $z=0$.

4.2. Solution for $t \gg 1$. The solution in this limit parallels that obtained above for the planar case in the limit $q \gg 1$. As previously mentioned, the temperature and the extent of the heated region continues to increase as time progresses. An orderof-magnitude analysis of (4.1) and (4.3) suggests the use of the modified variables 


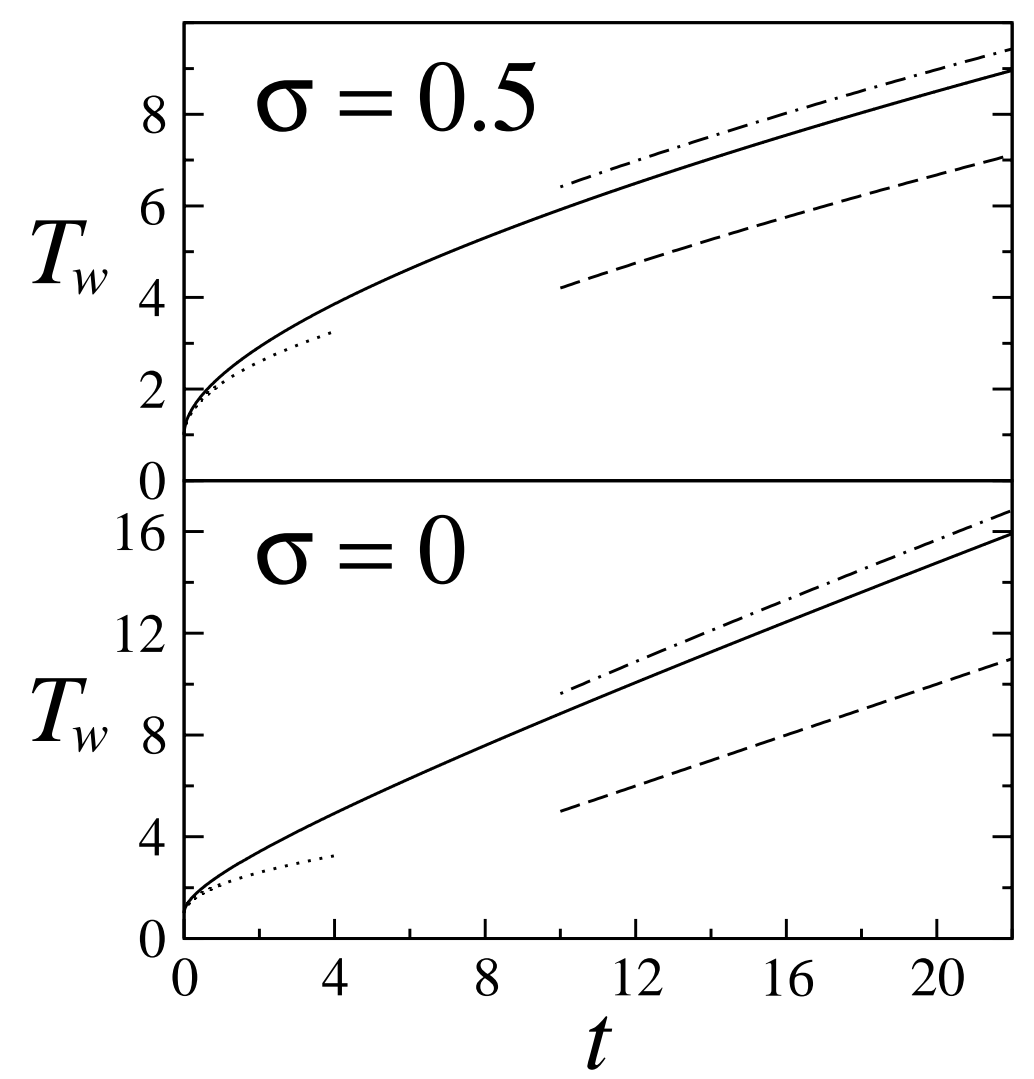

FIG. 6. The evolution with time of the wall temperature for different values of $\sigma$ obtained from numerical integration of (4.1) (solid lines), from the short-time prediction (4.8) (dotted lines), from the leading-order long-time prediction $T_{w}=\theta_{0}(0) t^{1 /(\sigma+1)}$ (dashed lines), and from the two-term expansion $T_{w}=t^{1 /(\sigma+1)}\left[\theta_{0}(0)+\theta_{1}(0) t^{-\mu_{0}}\right]$ (dot-dashed lines).

$\theta=T / t^{1 /(\sigma+1)}$ and $x=r / t$, of order unity, for the analysis of the limit $t \gg 1$, so that (4.1) and (4.3) take the form

$$
t \theta_{t}+\left(1+\theta^{\sigma} \theta_{x}-x\right) \theta_{x}+\frac{\theta}{\sigma+1}=\theta\left(\theta^{\sigma} \theta_{x}\right)_{x}
$$

and

$$
\left\{\begin{aligned}
x=0: & \theta^{\sigma} \theta_{x}=-1 \\
x=\infty: & \theta=1 / t^{1 /(\sigma+1)},
\end{aligned}\right.
$$

while the velocity is given by $v=1+\theta^{\sigma} \theta_{x}$. As can be seen, because of the rescaled variables employed in this limit, an additional convective term $-x \theta_{x}$ appears in (4.9), together with a damping term $\theta /(\sigma+1)$ associated with the growing temperature scale.

Introducing the expansion $\theta(x, t)=\theta_{0}(x)+t^{-\mu_{0}} \theta_{1}(x)+\cdots$ permits us to solve the problem in a sequential manner, with the unknown value of $\mu_{0}$ being determined as part of the asymptotic development as shown below. The function $\theta_{0}$ is obtained from

$$
\left(1+\theta_{0}^{\sigma} \theta_{0 x}-x\right) \theta_{0 x}+\frac{\theta_{0}}{\sigma+1}=\theta_{0}\left(\theta_{0}^{\sigma} \theta_{0 x}\right)_{x} \begin{cases}x=0: & \theta_{0}^{\sigma} \theta_{0 x}=-1 \\ x=1: & \theta_{0}=0\end{cases}
$$




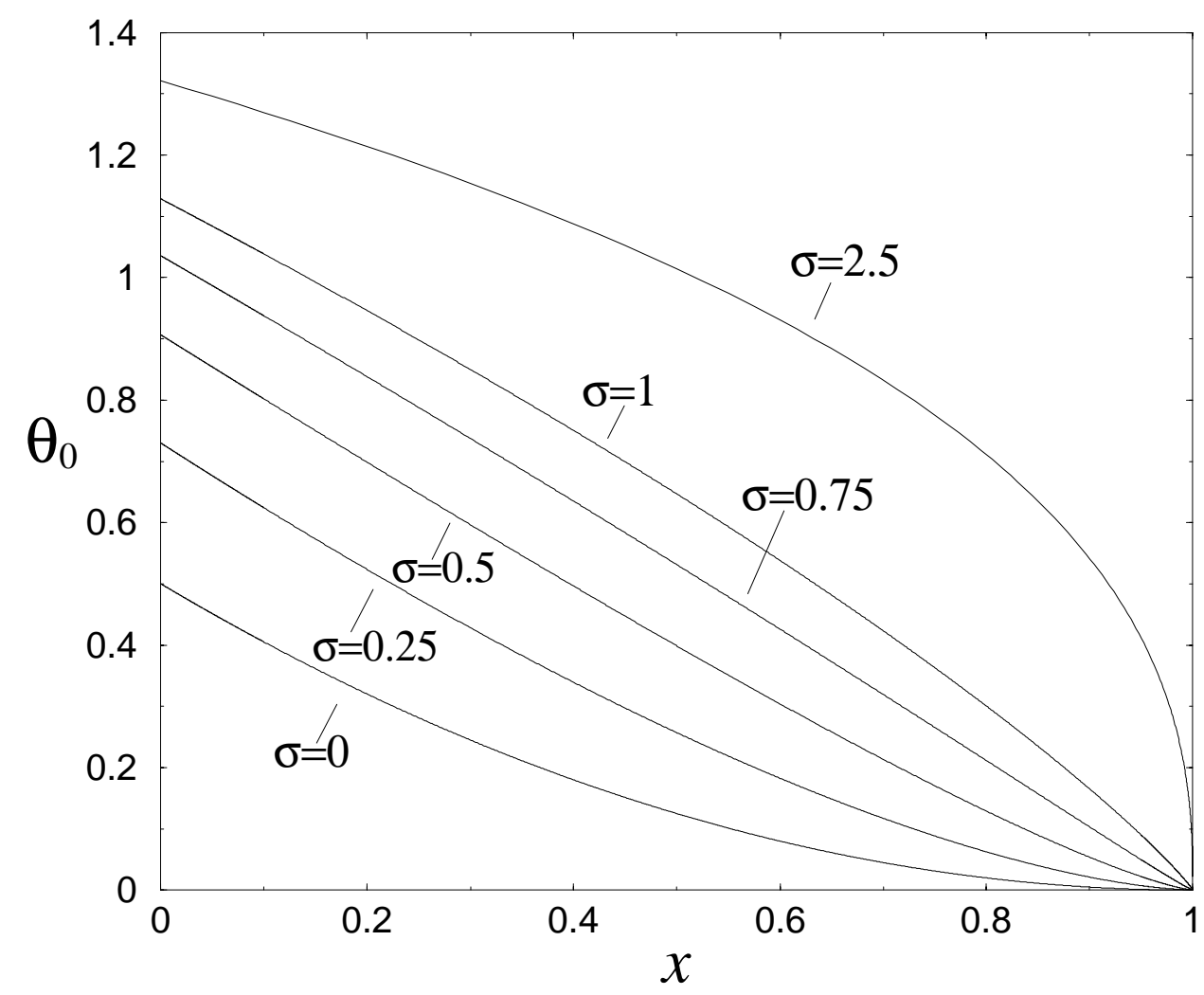

FIG. 7. The temperature profile $\theta_{0}$ obtained from (4.9) for different values of $\sigma$.

The problem (4.11) has a front solution, similar to that seen in the axisymmetric case for $q \gg 1$. Since both the heat-conduction term, $\theta_{0}\left(\theta_{0}^{\sigma} \theta_{0 x}\right)_{x}$, and the damping term, $\theta_{0} /(\sigma+1)$, vanish as the temperature approaches its zero boundary value, the front of the temperature distribution must lie at $x=1$, where the positive velocity due to thermal expansion, $v \simeq 1$, equals the apparent negative velocity $-x$.

When $\sigma=0$, the problem given in (4.11) has the exact solution (see [5])

$$
\left\{\begin{array}{lllr}
\theta_{0}=(1-x)^{2} / 2, & v=x, & \text { for } & 0<x<1 \\
\theta_{0}=0, & v=1, & \text { for } & 1<x
\end{array}\right.
$$

Another exact solution appears when $\sigma=1$, as can be seen by rewriting (4.5) in terms of the intermediate coordinate $\bar{z}=z /\left(2 t^{1 / 2}\right)$ to give the implicit representation [5]

$$
\theta_{0}=2 \mathrm{i}^{1} \operatorname{erfc}(\bar{z}) \text { and } x=1-4 \mathrm{i}^{2} \operatorname{erfc}(\bar{z}) .
$$

Numerical integration is necessary to compute profiles of $\theta_{0}$ when $\sigma \neq(0,1)$. The temperature profiles (4.12) and (4.13) are shown in Figure 7 along with the numerical results corresponding to $\sigma=(0.25,0.5,0.75,2.5)$. A shooting technique started at $x=$ 0 was used for the integration of (4.11), with $\theta(0)$ utilized as the shooting parameter to be varied in the iteration procedure. This initial value, which equals $\theta_{0}(0)=0.5$ for $\sigma=0, \theta_{0}(0)=2 / \pi^{1 / 2}$ for $\sigma=1$, and $\theta_{0}(0)=(0.7298,0.9063,1.0356,1.3212)$ for $\sigma=$ $(0.25,0.5,0.75,2.5)$, determines the leading-order prediction for the wall temperature 
$T_{w}=\theta_{0}(0) t^{1 /(\sigma+1)}$. The comparisons with the results of the numerical integrations for large times, shown in Figure 6, clearly indicate that the asymptotic description must be carried on to the following order for increased accuracy.

This first-order correction $\theta_{1}(x)$ must satisfy the conservation equation (4.9) linearized about $\theta_{0}$

$$
\begin{aligned}
& \left(\frac{1}{\sigma+1}-\mu_{0}\right) \theta_{1}+(1-x) \theta_{1 x} \\
& \quad=\theta_{0}^{\sigma}\left\{\left[(\sigma+1) \theta_{0 x x}-\sigma(1-\sigma) \frac{\theta_{0 x}^{2}}{\theta_{0}}\right] \theta_{1}-2(1-\sigma) \theta_{0 x} \theta_{1 x}+\theta_{0} \theta_{1 x x}\right\},
\end{aligned}
$$

subject to the boundary conditions

$$
x=0: \quad \theta_{0}^{1+\sigma} \theta_{1 x}-\sigma \theta_{1}=0
$$

and

$$
x=1: \quad \theta_{1}=0 .
$$

As occurred before with the perturbation problem (3.11)-(3.13), for each value of $\mu_{0}$ the problem (4.14)-(4.16) admits a single nontrivial solution that can be determined aside from an arbitrary multiplicative factor. The value of $\mu_{0}$ is determined from matching the first two terms of the high-temperature distribution $\theta=\theta_{0}+t^{-1 /(\sigma+1)} \theta_{1}$ with the leading-order temperature representation across the corner layer, located around $x=1$. It is remarkable that the first-order correction can be determined without taking into account the initial non-self-similar growth period, thereby indicating that memory effects emerge in the asymptotic development for large times only at higher orders. The associated corrections should be computed from matching the asymptotic results with the numerical computations for $t \sim 1$, a development not pursued further here.

As seen before for the line source, the structure of the solution for $\sigma=1$ and $\sigma>1$ is different from that encountered with $\sigma<1$. The analyses of the former solutions, which are given in the appendixes, reveal that $\mu_{0}=1 /(\sigma+1)$, corresponding to a correction in temperature $T$ of order unity. The corrections are larger when $\sigma<1$, when the leading-order temperature profile approaches the boundary $x=1$ according to the local description

$$
\theta_{0}=\left(\frac{(1+\sigma)}{2(1-\sigma)}\right)^{1 /(\sigma+1)}(1-x)^{2 /(\sigma+1)}
$$

as can be obtained from (4.11). The corner layer, where the temperature $T$ is of order unity, corresponds to distances $(1-x)$ of order $t^{-1}$. Introducing the coordinate $\chi=(t / 2)^{1 / 2}(1-r / t)$ reduces the leading-order problem to that given in (3.14), whose solution matches asymptotically with the boundary distribution (4.17). Furthermore, inspection of (3.15) indicates that the order of the first-order correction must be

$$
\mu_{0}=\frac{\sigma+\sqrt{2-\sigma^{2}}}{2(1+\sigma)}
$$

to complete the matching, and that

$$
x=1: \quad \theta_{1}=A[(1-x) / \sqrt{2}]^{\left(2-\sigma-\sqrt{2-\sigma^{2}} /(1+\sigma)\right.}
$$


must replace (4.16) to provide uniqueness for the solution to (4.14). In general, numerical integration is required to compute $\theta_{1}$, the only exception being the case $\sigma=0$, for which the exact solution

$$
\theta_{1}=\left(\frac{A}{2^{1-1 / \sqrt{2}}}\right)\left[(1-x)^{2-\sqrt{2}}-\frac{2-\sqrt{2}}{1+\sqrt{2}}(1-x)^{1+\sqrt{2}}\right]
$$

is available.

The results of the asymptotic analysis can be combined to give a uniformly valid description for the temperature. The corresponding composite expansion is that given in (3.18), with $q, \xi$, and $\mu_{1}$ being replaced with $t, x$, and $\mu_{0}$, and with the origin for the Heaviside function being $x=1$. The resulting temperature profile and its accompanying velocity profile are plotted in Figure 5, showing good agreement for the value $t=22$ considered. The relatively small errors observed, of order unity, correspond to a correction at the following order in the asymptotic analysis, which is not computed here. As seen in Figure 6, an error of order unity is also present in the second-order asymptotic prediction for the wall temperature $T_{w}=t^{1 /(\sigma+1)}\left(\theta_{0}(0)+\right.$ $\left.\theta_{1}(0) t^{-\mu_{0}}\right)$, where $\theta_{1}(0)=(2.359,2.223,1.927,1.486)$ for $\sigma=(0,0.25,0.5,0.75)$.

5. The point source of heat. The characteristic scales for this problem, defined in order of magnitude in (1.3) and (1.4), were used to define the dimensionless variables $t=\alpha_{o}\left[\mathrm{q}_{2} /\left(4 \pi k_{o} T_{o}\right)\right]^{-2} \mathrm{t}, r=\mathrm{r} /\left[\mathrm{q}_{2} /\left(4 \pi k_{o} T_{o}\right)\right]$, and $v=\mathrm{v}\left[\mathrm{q}_{2} /\left(4 \pi k_{o} T_{o}\right)\right] / \alpha_{o}$. The temperature $T$ is determined by integrating

$$
\frac{\partial T}{\partial t}-\frac{T^{2}}{r^{2}} \frac{\partial}{\partial r}\left[\frac{1}{T}\left(1+T^{\sigma} r^{2} \frac{\partial T}{\partial r}\right)\right]=0
$$

with initial condition

$$
t=0, \quad 0 \leq r<\infty: T=1
$$

and boundary conditions

$$
t>0 \begin{cases}r=0: & r^{2} T^{\sigma} \partial T / \partial r=-1 \\ r=\infty: & T=1\end{cases}
$$

while the velocity can be computed from

$$
v=\frac{1}{r^{2}}\left(1+r^{2} T^{\sigma} \frac{\partial T}{\partial r}\right)
$$

As in the planar case, $\sigma$ remains as the only parameter left in the problem. Because of the boundary condition at $r=0$, the temperature profile presents an infinite value at the point source for $t>0$. Hence, the numerical integration of the problem (5.1)-(5.3) must account for the singular character of the solution near the origin, where

$$
T^{\sigma+1}=\frac{(\sigma+1)}{r}+C(t)
$$

In particular, the initial profile $T=1$ must be replaced with the leading-order representation emerging for $t \ll 1$. 
5.1. Initial temperature growth. As previously anticipated, the structure of the solution in this limit $t \ll 1$ is that found for $q \gg 1$ in the axisymmetric case and for $t \gg 1$ in the planar case, that is, a neatly defined central region of high temperature separated from the outer cold gas at temperature $T=T_{o}$ by a thin corner layer of warm fluid. The appropriate scales for length and temperature to describe the hot region, $t^{1 / 3}$ and $t^{-1 /[3(\sigma+1)]}$, can be anticipated from the balance of the three terms in (5.1). Correspondingly, the associated rescaled variables $\theta=t^{1 /[3(\sigma+1)]} T$ and $y=r / t^{1 / 3}$ reduce (5.1) and (5.3) to

$$
t \theta_{t}+\left[y^{-2}\left(1+y^{2} \theta^{\sigma} \theta_{y}\right)-\frac{y}{3}\right] \theta_{y}-\frac{\theta}{3(\sigma+1)}=\theta y^{-2}\left(y^{2} \theta^{\sigma} \theta_{y}\right)_{y}
$$

and

$$
\left\{\begin{aligned}
y=0: & y^{2} \theta^{\sigma} \theta_{y}=-1 \\
y=\infty: & \theta=t^{1 /[3(\sigma+1)]}
\end{aligned}\right.
$$

Because of the growing length scale that has been introduced, besides the rescaled thermal-expansion velocity of order unity, $t^{2 / 3} v=y^{-2}\left(1+y^{2} \theta^{\sigma} \theta_{y}\right)$, there exists in (5.6) a negative apparent velocity $-y / 3$. Similarly, the decreasing scale used for the temperature leads to the negative damping term $-\theta /[3(\sigma+1)]$.

Introducing the expansion $\theta(y, t)=\theta_{0}(y)+t^{\mu_{2}} \theta_{1}(y)+\cdots$ yields at leading order

$$
\left[y^{-2}\left(1+y^{2} \theta_{0}^{\sigma} \theta_{0 y}\right)-\frac{y}{3}\right] \theta_{0 y}-\frac{\theta_{0}}{3(\sigma+1)}=\theta_{0} y^{-2}\left(y^{2} \theta_{0}^{\sigma} \theta_{0 y}\right)_{y}
$$

to be integrated with boundary conditions

$$
\left\{\begin{aligned}
y=0: & y^{2} \theta_{0}^{\sigma} \theta_{0 y}=-1 \\
y=3^{1 / 3}: & \theta_{0}=0
\end{aligned}\right.
$$

As before, the balance between the thermal-expansion velocity and the apparent velocity determines the location of the front $y=3^{1 / 3}$. A shooting method was used to integrate (5.8). Integration was initiated near $y=0$, where the temperature profile is of the form $\theta_{0}^{\sigma+1}=(\sigma+1) / y+c$. The unknown shooting parameter $c$ was varied in the numerical integration to satisfy the boundary condition at $y=3^{1 / 3}$, yielding the profiles shown in Figure 8. The negative constant $c=-(0.8390,1.2173,1.5806,2.6397)$ for $\sigma=(0,0.5 .1 .0,2.5)$ provides $C=c t^{-1 / 3}$ for the initial evolution of the constant $C$ in $(5.5)$.

If $\sigma<1$, the function $\theta_{0}$ is seen to approach the boundary according to

$$
\theta_{0}=\left(\frac{7(1+\sigma)}{6(1-\sigma)}\right)^{1 /(1+\sigma)}\left(3^{1 / 3}-y\right)^{2 /(1+\sigma)}
$$

to be matched with the temperature profile across the transition layer, which is determined at leading order by (3.14), with the similarity coordinate being defined as $\chi=t^{-1 / 6}(6 / 7)^{1 / 2}\left(3^{1 / 3}-r / t^{1 / 3}\right)$. Matching the first two terms in the expansion for $\theta$ with (3.15) yields in this case

$$
\mu_{2}=\frac{\sigma+\sqrt{2-\sigma^{2}}}{6(1+\sigma)}
$$




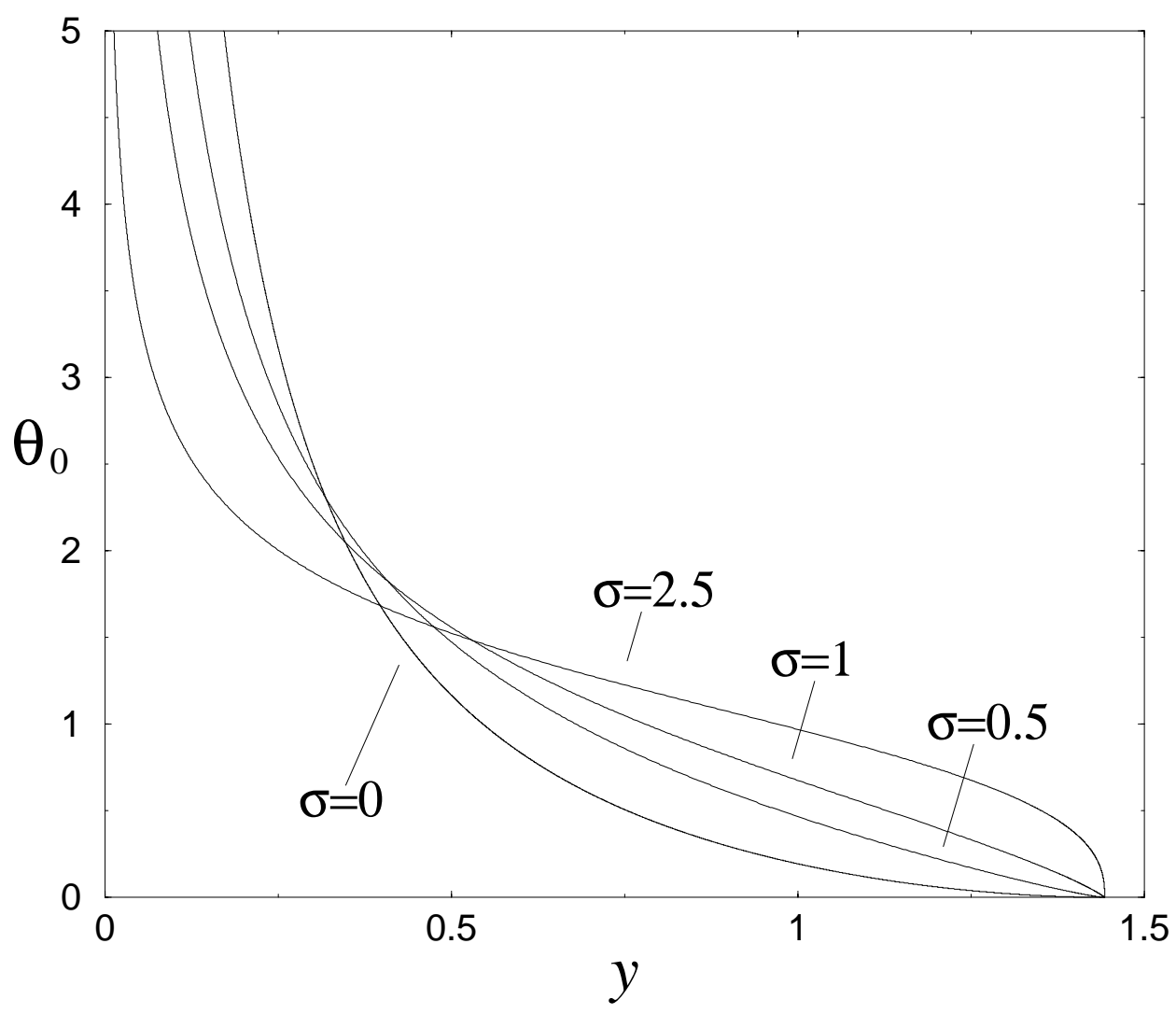

FIG. 8. The temperature profiles obtained by integration of (5.8) for $\sigma=(0,0.5,1.0,2.5)$.

along with the asymptotic value

$$
\theta_{1} \rightarrow A\left[(7 / 6)^{1 / 2}\left(3^{1 / 3}-y\right)\right]^{\left(2-\sigma-\sqrt{2-\sigma^{2}}\right) /(1+\sigma)}
$$

to be used as a boundary condition at $y=3^{1 / 3}$ when computing the first-order correction $\theta_{1}$. The singular case $\sigma=1$, when

$$
\theta_{0}=\left(3^{1 / 3}-y\right)\left[\frac{7}{3} \ln \left(\frac{1}{3^{1 / 3}-y}\right)\right]^{1 / 2}
$$

for $0<3^{1 / 3}-y \ll 1$, is described separately in Appendix A, while the the case $\sigma>1$, when $\theta_{0} \propto\left(3^{1 / 3}-y\right)^{1 / \sigma}$ near the boundary, is described in Appendix B.

5.2. Temperature and velocity distributions. As previously mentioned, the results of the asymptotic analysis for $t \ll 1$ were employed to enable integrations of (5.1). The two-term expansion $\theta=\theta_{0}+t^{\mu_{2}} \theta_{1}$ was combined with the solution in the corner layer to provide the corresponding composite expansion, which is that given in (3.18) with the exponents $-1 /[3(\sigma+1)]$ and $\mu_{2}$ and the variables $y$ and $t$ replacing $1 /(\sigma+1),-\mu_{1}, \xi$, and $q$, respectively, and with the origin for the Heaviside function being $y=3^{1 / 3}$. This composite expansion evaluated at $t=0.01$ was used as an initial condition in the integrations shown in Figure 9, where the temperature 


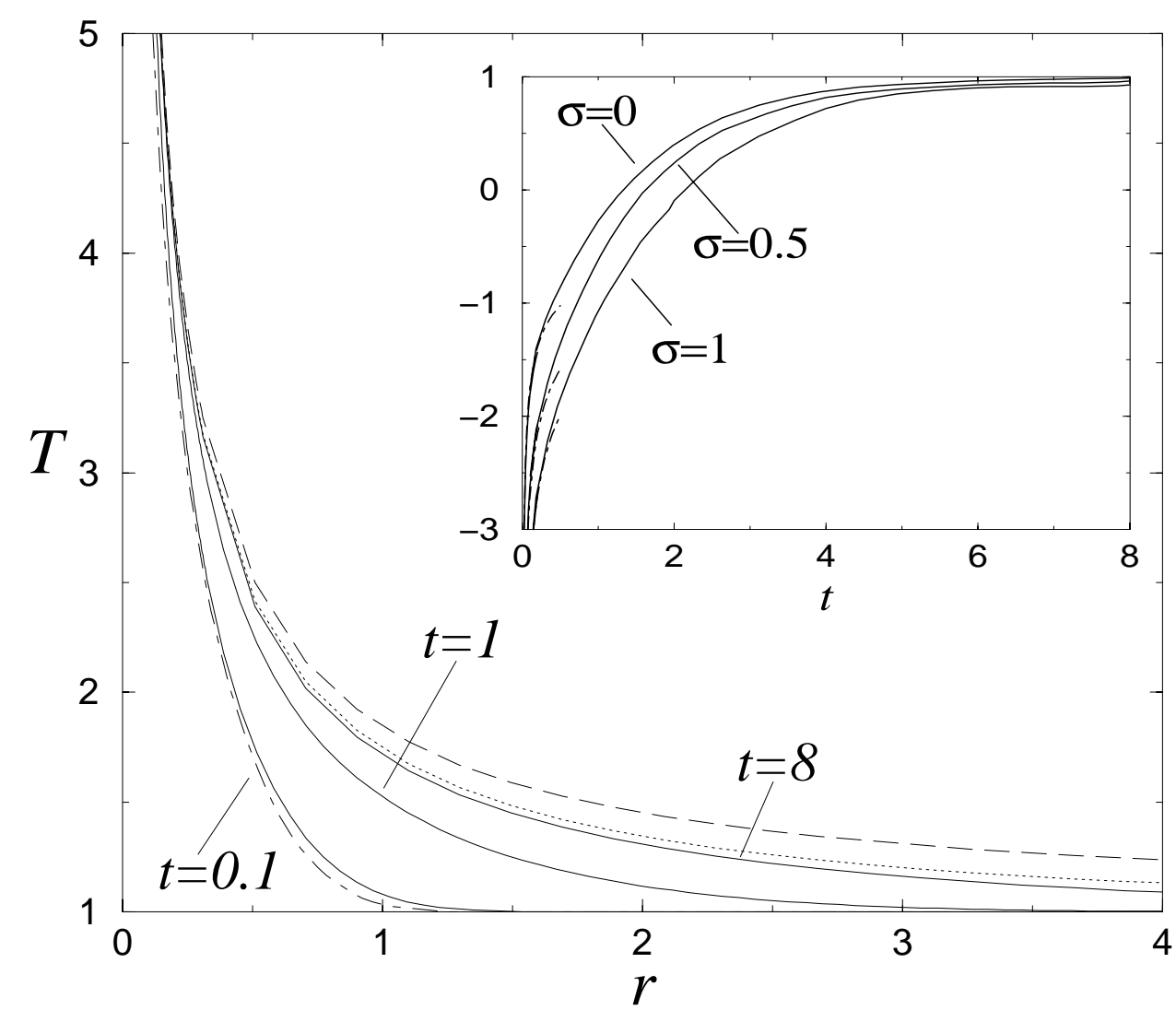

FIG. 9. The temperature profile corresponding to the final steady solution (5.14) with $\sigma=$ 0.5 (dashed line), along with those obtained by integration of (5.1) (solid lines), from the shorttime composite expansion (dot-dashed line), and from the long-time quasi-steady expression (5.16) (dotted line); the inset shows the variation of the constant $C$ together with the short-time prediction $C=c t^{-1 / 3}$ (dot-dashed lines).

profiles corresponding to $t=(0.1,1.0,8.0)$ are shown. As can be observed, the comparison with the short-time composite expansion for $t=0.1$ still gives reasonably good agreement. For completeness, the plot exhibits in an inset the variation with time of the constant $C$ corresponding to the near-origin temperature distribution (5.5), along with the short-time prediction $C=c t^{-1 / 3}$.

The temperature profiles can be used in (5.4) to provide the associated velocity profiles, which are shown in Figure 10. The solution is seen to evolve rapidly from the initial large velocities of order $t^{-2 / 3}$ to the final quasi-stagnant solution corresponding to $t \gg 1$, which is described below.

5.3. Quasi-steady long-time solution. For asymptotically large values of $t$, the solution evolves to approach the profile

$$
T_{s}=\left(1+\frac{\sigma+1}{r}\right)^{1 /(\sigma+1)} .
$$

This steady solution (5.14) and its associated velocity field $v=0$ are correct to all algebraic orders at distances $r$ of order unity; i.e., the investigation in the limit $t \gg 1$ 


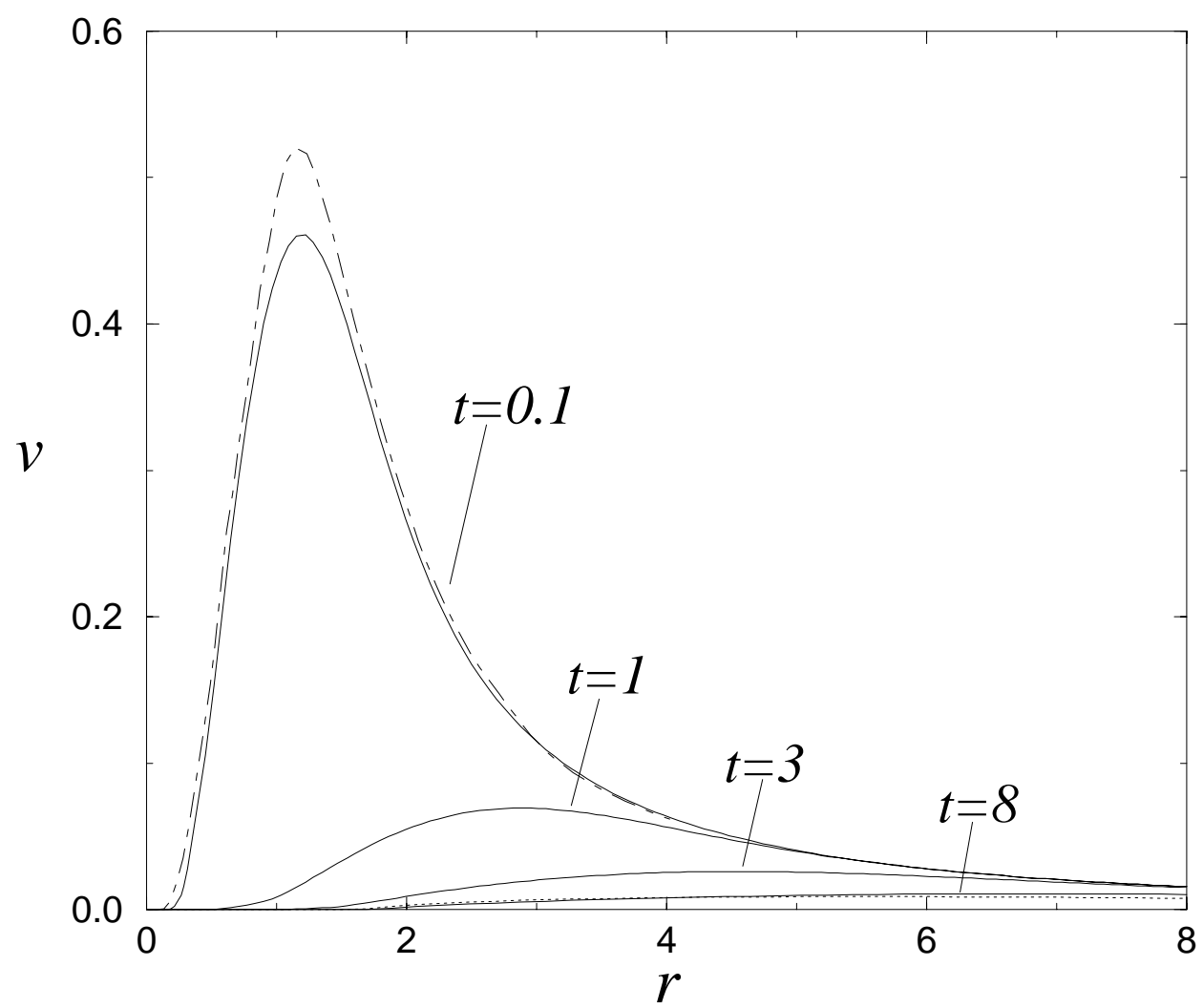

FIG. 10. The velocity profiles obtained by evaluating (5.4) with the numerical temperature profile (solid lines) and with the short-time composite expansion (dot-dashed lines). The dotted line represents the long-time quasi-steady solution (5.17).

of perturbations of the form $T=T_{s}(r)+t^{-\alpha} T_{\alpha}(r)$ yields $T_{\alpha}=0$ irrespective of the value of $\alpha$.

Unsteady effects are seen to enter farther from the heat source, in a far-field region corresponding to distances of order $t^{1 / 2}$ where only small temperature increments $T-1$ of order $t^{-1 / 2}$ exist. To study this far-field region, it is convenient to employ the similarity coordinate $\eta=r / t^{1 / 2}$, along with the rescaled temperature increment $T-1=t^{-1 / 2} \Theta$, where an expansion of the form $\Theta(\eta, t)=\Theta_{0}(\eta)+t^{-1 / 2} \Theta_{1}(\eta)+\cdots$ is assumed. Introducing these new variables into (5.1) yields at leading order

$$
\left(\eta^{2} / 2\right)\left(\Theta_{0}+\eta \Theta_{0 \eta}\right)+\left(\eta^{2} \Theta_{0 \eta}\right)_{\eta}\left\{\begin{array}{cl}
\eta \rightarrow 0: & \Theta_{0}=1 / \eta \\
\eta \rightarrow \infty: & \Theta_{0}=0
\end{array}\right.
$$

where the boundary condition as $\eta \rightarrow 0$ comes from matching with the steady solution (5.14). Straightforward integration gives $\Theta_{0}=\eta^{-1} \operatorname{erfc}(\eta / 2)$. Now combining the inner steady-state profile with the far-field transient solution provides the composite expansion $T=T_{s}+t^{-1 / 2}\left(\Theta_{0}-1 / \eta\right)$, which gives the solution for the large-time temperature evolution with errors of order $t^{-1}$. At the same level of approximation, one may write

$$
T=\left[1+\frac{\sigma+1}{r} \operatorname{erfc}\left(\frac{r}{2 t^{1 / 2}}\right)\right]^{1 /(\sigma+1)}
$$


for the temperature profile, a compact expression that can be used in (5.4) to obtain the velocity profile

$$
v=\frac{1}{r^{2}}\left[\operatorname{erf}\left(\frac{r}{2 t^{1 / 2}}\right)-\frac{r}{(\pi t)^{1 / 2}} \exp \left(\frac{-r^{2}}{4 t}\right)\right]
$$

Note that, when $\sigma=0,(5.16)$ corresponds to the exact self-similar solution achieved with constant density and constant conductivity [4]. These large-time predictions are compared in Figures 9 and 10 with results of numerical integrations for $t=8$, yielding reasonably good agreement.

6. Conclusions. The transient, one-dimensional, near-isobaric, buoyancy-free flow field induced by a localized energy source of constant rate has been analyzed for planar, cylindrical, and spherical geometries. The convection induced by thermal expansion is seen to aid the transport of heat away from the source, in a nonlinear process of evolution that has been computed with account taken of the variable thermal conductivity typical of gases. Our study shows a self-similar solution for the line source, with the dimensionless heat release $q$ entering as a parameter, while both the planar source and the point source require consideration of a nonlinear parabolic equation for the time evolution of the temperature.

The analysis reveals that front solutions emerge when the resulting temperatures become much larger than the initial temperature, with the front location being determined a priori from a convective balance. It is shown that the inner structure of the planar thin front, which is identical for all three geometrical configurations, determines the first-order correction in the hot region. Note that front solutions can also be expected to emerge as limiting solutions when a variable heating rate $\mathrm{q}_{j}(\mathrm{t})$ is applied, a problem to be addressed in future work. In that case, unsteady effects are likely to emerge in the hot region at leading order, while the inner structure of the thin front is expected to evolve in a quasi-steady manner. Also of interest is the investigation of the effect of compressibility on the heat propagation process from point and line sources, as done for planar sources by Clarke, Kassoy, and Riley [6]. Future research should also consider the solution emerging after the heat source is switched off. A related study is that of Meerson [12], who considered the conductive cooling of a gas heated by a localized deposition of heat.

The quantitative information provided here can be of interest, for instance, in analyses of ignition processes of a reactive gas mixture by localized energy sources [10]. The corresponding energy conservation equation should incorporate a heatrelease term, and should be supplemented by conservation equations for the chemical species. The ignition process typically involves an initial quasi-frozen period with negligible chemical heat release, in which the description given here holds, followed by a period of significant exothermicity. For instance, for ignition of hydrogen-oxygen mixtures $[10,11]$, the initial branched-chain explosion $[3,14]$ produced after the heat source is turned on could be computed with the temperature and velocity fields given above. It can be anticipated that, since ignition often requires temperatures that are much larger than the normal ambient value, the front solutions described above will be particularly useful for these ignition studies.

Appendix A. The front solution for $\sigma=1$. The structure of the front when $\sigma=1$ is different from that described in the text for $\sigma$ in the range $0 \leq \sigma<1$. We give first the solution corresponding to the line source of heat, and describe later the small modifications required for the planar and spherical geometries. 
To construct the solution one needs to match the leading-order solution across the corner layer with the the two-term expansion $\theta=\theta_{0}+q^{-1 / 2} \theta_{1}$, where we already anticipate that the order of the correction is $\mu_{1}=1 / 2$. The first-order correction $\theta_{1}$ is determined by integrating

$$
\left(1-\frac{\xi^{2}}{2}\right) \theta_{1 \xi}=2 \theta_{0}\left(\xi \theta_{0 \xi}\right)_{\xi} \theta_{1}+\theta_{0}^{2}\left(\xi \theta_{1 \xi}\right)_{\xi}
$$

with boundary conditions

$$
\begin{cases}\xi=0: & \left(\theta_{0} \theta_{1}\right)_{\xi}=0 \\ \xi=\sqrt{2}: & \theta_{1}=2 D \ln [1 /(\sqrt{2}-\xi)]\end{cases}
$$

where $D$ is an unknown constant to be determined as part of the matching procedure. Near the front, the two-term expansion $\theta=\theta_{0}+q^{-1 / 2} \theta_{1}$ can be written as

$$
\theta=\sqrt{2}(\sqrt{2}-\xi)\left[\ln \left(\frac{1}{\sqrt{2}-\xi}\right)\right]^{1 / 2}+q^{-1 / 2} 2 D \ln \left(\frac{1}{\sqrt{2}-\xi}\right)
$$

where use has been made of (3.9).

Observation of (A.3) reveals that the corner layer, where the temperature becomes of order unity, is a factor $(\ln q)^{-1 / 2}$ thinner than that found with $\sigma<1$, and is displaced towards the cold outer gas. More precisely, the front extends over distances of order $q^{-1 / 2}(\ln q)^{-1 / 2}$ around $\xi=\sqrt{2}+D q^{-1 / 2}(\ln q)^{1 / 2}$, where $D$ is the unknown constant appearing in (A.2). The appropriate inner coordinate must incorporate both a translation and a dilatation according to $\zeta=q^{1 / 2}(\ln q)^{1 / 2}\left[\sqrt{2}+D q^{-1 / 2}(\ln q)^{1 / 2}-\xi\right]$. The problem reduces to that of integrating

$$
T^{2} T_{\zeta \zeta}-D T_{\zeta}=0 \begin{cases}\zeta \rightarrow-\infty: & T-1 \rightarrow 0 \\ \zeta \rightarrow \infty: & T_{\zeta}-1 \rightarrow 0\end{cases}
$$

where the boundary condition as $\zeta \rightarrow \infty$ comes from matching with (A.3). Integrating once with use made of the boundary condition $T(-\infty)=1$ yields $T_{\zeta}=D(1-1 / T)$, whereas imposing the linear profile on the hot boundary finally determines $D=1$. This value can be used in (A.2) to complete the boundary conditions necessary to uniquely determine the first-order perturbation $\theta_{1}$. Note that the second quadrature for the corner-layer equation, $T+\ln (T-1)=\zeta+\zeta_{o}$, contains an arbitrary translation $\zeta_{o}$, which could be computed from higher-order terms in the asymptotic expansion.

The solutions encountered for $j=0$ and $j=2$ also respond to the same structure. Thus, for the planar heat source, the first-order perturbation $\theta_{1}$ in the expansion $\theta=\theta_{0}+t^{-1 / 2} \theta_{1}$ is determined from

$$
(1-x) \theta_{1 x}=2 \theta_{0} \theta_{0 x x} \theta_{1}+\theta_{0}^{2} \theta_{1 x x} \begin{cases}x=0: & \left(\theta_{0} \theta_{1}\right)_{x}=0, \\ x=1: & \theta_{1}=2 D \ln [1 /(1-x)],\end{cases}
$$

while for the point source the expansion in the hot region becomes $\theta=\theta_{0}+t^{1 / 6} \theta_{1}$, where $\theta_{1}$ satisfies

$$
\left(1-\frac{y^{3}}{3}\right) \theta_{1 y}=2 \theta_{0}\left(y^{2} \theta_{0 y}\right)_{y} \theta_{1}+\theta_{0}^{2}\left(y^{2} \theta_{1 y}\right)_{y}
$$

with boundary conditions

$$
\begin{cases}y=0: & \left(\theta_{0} \theta_{1}\right)_{y}=0 \\ y=3^{1 / 3}: & \theta_{1}=6 D \ln \left[1 /\left(3^{1 / 3}-y\right)\right]\end{cases}
$$


On the other hand, the inner coordinates $\zeta=(t / 2)^{1 / 2}(\ln t)^{1 / 2}\left[1+D(t / 2)^{-1 / 2}(\ln t)^{1 / 2}-\right.$ $x]$ for $j=0$, and $\zeta=[(7 / 2) \ln (1 / t)]^{1 / 2} t^{-1 / 6}\left\{3^{1 / 3}+[(7 / 2) \ln (1 / t)]^{1 / 2} t^{1 / 6}(D / 3)-y\right\}$ for $j=2$, reduce the corner-layer problem to (A.4), indicating that $D=1$ should be used in (A.5) and (A.7).

Appendix B. The front solution for $\sigma>1$. The structure of the thermal wave near the edge for $\sigma>1$ is similar to that described above for $\sigma=1$. As explained in the text, the asymptotic behavior of the leading-order profile $\theta_{0}$ near the edge is

$$
\theta_{0}=E\left[(j+1)^{1 /(j+1)}-\xi\right]^{1 / \sigma},
$$

where $E$ is a constant to be determined from the numerical integration, and where $\xi$ should be replaced with $x$ and $y$ for $j=0$ and $j=2$, respectively, following the notation used in the text.

As before, we shall first give the solution corresponding to $j=1$, for which we assume the expansion $\theta=\theta_{0}+q^{-1 /(\sigma+1)} \theta_{1}$. The first-order correction $\theta_{1}$ can be calculated by integrating

$$
\left(1-\frac{\xi^{2}}{2}\right) \theta_{1 \xi}=2 \theta_{0}\left(\xi \theta_{0}^{\sigma-1} \theta_{0 \xi}\right)_{\xi} \theta_{1}+\theta_{0}^{2}\left(\xi \theta_{0}^{\sigma-1} \theta_{1 \xi}\right)_{\xi}+(\sigma-1) \theta_{0}^{2}\left(\xi \theta_{0}^{\sigma-2} \theta_{0 \xi} \theta_{1}\right)_{\xi}
$$

with boundary conditions

$$
\left\{\begin{array}{l}
\xi=0: \theta_{0} \theta_{1 \xi}+\sigma \theta_{1} \theta_{0 \xi}=0 \\
\xi=\sqrt{2}: \theta_{1}=D(\sqrt{2}-\xi)^{(1-\sigma) / \sigma}
\end{array}\right.
$$

where $D$ is a constant to be determined below. Near the edge, the two-term expansion for $\theta$ gives

$$
\theta=E(\sqrt{2}-\xi)^{1 / \sigma}+D(\sqrt{2}-\xi)^{(1-\sigma) / \sigma} .
$$

As seen before for $\sigma=1$, the corner layer that appears is thinner than that corresponding to $\sigma<1$ and is displaced towards the outer cold gas. Its inner structure can be described by introducing the variable $\zeta=(\sigma / E) q^{\sigma /(\sigma+1)}\left[\sqrt{2}+(\sigma / E) D q^{-1 /(\sigma+1)}-\xi\right]$ to yield the problem

$$
T^{2}\left(T^{\sigma-1} T_{\zeta}\right)_{\zeta}-D T_{\zeta}=0\left\{\begin{array}{l}
\zeta \rightarrow-\infty: T \rightarrow 1 \\
\zeta \rightarrow \infty: T_{\zeta} \rightarrow 1 / \sigma \zeta^{(1-\sigma) / \sigma}
\end{array}\right.
$$

The boundary condition as $\zeta \rightarrow-\infty$ can be used in a first quadrature to give us $D\left[(T-1) / T^{\sigma}\right]=T_{\zeta}$, which can be evaluated as $\zeta \rightarrow \infty$ to provide $D=1 / \sigma$ for the value of the unknown constant $D$.

The same structure appears near the edge of the thermal wave when $j=0$ and $j=$ 2. For the planar case, the first-order correction in the expansion $\theta=\theta_{0}+t^{-1 /(\sigma+1)} \theta_{1}$ is determined from

$$
(1-x) \theta_{1 x}=2 \theta_{0}\left(\theta_{0}^{\sigma-1} \theta_{0 x}\right)_{x} \theta_{1}+\theta_{0}^{2}\left(\theta_{0}^{\sigma-1} \theta_{1 x}\right)_{x}+(\sigma-1) \theta_{0}^{2}\left(\theta_{0}^{\sigma-2} \theta_{0 x} \theta_{1}\right)_{x},
$$

with boundary conditions

$$
\left\{\begin{array}{l}
x=0: \theta_{0} \theta_{1 x}+\sigma \theta_{1} \theta_{0 x}=0 \\
x=1: \theta_{1}=D(1-x)^{(1-\sigma) / \sigma}
\end{array} .\right.
$$


Similarly, the expansion for $j=2$ is $\theta=\theta_{0}+t^{1 /[3(\sigma+1)]} \theta_{1}$, where $\theta_{1}$ is computed by integrating

$$
\left(1-\frac{y^{3}}{3}\right) \theta_{1 y}=2 \theta_{0}\left(y^{2} \theta_{0}^{\sigma-1} \theta_{0 y}\right)_{y} \theta_{1}+\theta_{0}^{2}\left(y^{2} \theta_{0}^{\sigma-1} \theta_{1 y}\right)_{y}+(\sigma-1) \theta_{0}^{2}\left(y^{2} \theta_{0}^{\sigma-2} \theta_{0 y} \theta_{1}\right)_{y},
$$

with boundary conditions

$$
\left\{\begin{array}{l}
y=0: \theta_{0} \theta_{1 y}+\sigma \theta_{1} \theta_{0 y}=0 \\
y=3^{1 / 3}: \theta_{1}=3 D\left(3^{1 / 3}-y\right)^{(1-\sigma) / \sigma} .
\end{array}\right.
$$

Use of the inner coordinates $\zeta=(\sigma / E)(t / 2)^{\sigma /(\sigma+1)}\left[1+(\sigma / E) D(t / 2)^{-1 /(\sigma+1)}-x\right]$ for

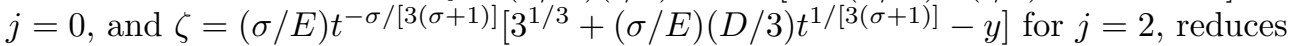
the description of the corner layer to the problem given in (B.5), so that the value $D=1 / \sigma$ is obtained for the constant $D$ appearing in (B.7) and (B.9).

\section{REFERENCES}

[1] M. Abramowitz And A. Stegun, Handbook of Mathematical Functions, Dover, New York, 1965.

[2] D. G. Aronson, Regularity of flows in porous media: A survey, in Nonlinear Diffusion Equations and Their Equilibrium States, W. M. Ni, L. A. Peletier, and J. Serrin, eds., SpringerVerlag, New York, 1988, pp. 35-49.

[3] L. L. Bonilla, A. L. SÁnChez, AND M. CARREtero, The description of homogeneous branchedchain explosions with slow radical recombination by self-adjusting time scales, SIAM J. Appl. Math., 61 (2000), pp. 528-550.

[4] H. S. Carslaw and J. C. Jaeger, Conduction of Heat in Solids, 2nd ed., Oxford University Press, London, 1959.

[5] J. J. Clarke, D. R. Kassoy, And N. RiLey, Shocks generated in a confined gas due to rapid heat addition at the boundary. I. Weak shock waves, Proc. Roy. Soc. London A, 393 (1984), pp. 309-329.

[6] J. J. Clarke, D. R. Kassoy, And N. Riley, Shocks generated in a confined gas due to rapid heat addition at the boundary. II. Strong shock waves, Proc. Roy. Soc. London A, 393 (1984), pp. 331-351.

[7] J. Crank, The Mathematics of Diffusion, 2nd ed., Clarendon Press, Oxford, UK, 1975.

[8] V. N. KurdYumov AND A. Liñ́n, Free convection from a point source of heat, and heat transfer from spheres at small Grashof numbers, Int. J. Heat Mass Transfer, 42 (1999), pp. 3849-3860.

[9] A. LiñÁn And V. N. Kurdyumov, Laminar free convection induced by a line heat source, and heat transfer from wires at small Grashof numbers, J. Fluid Mech., 362 (1998), pp. 199-227.

[10] U. MAAS AND J. WARNATZ, Ignition processes in hydrogen-air mixtures, Combustion and Flame, 74 (1988), pp. 53-69.

[11] U. MaAs, B. Raffel, J. Wolfrum, and J. Warnatz, Observation and simulation of laser induced ignition processes in $\mathrm{O}_{2}-\mathrm{O}_{3}$ and $\mathrm{H}_{2}-\mathrm{O}_{2}$ mixtures, Proc. Comb. Institute, 21 (1986), pp. 1869-1876.

[12] B. MeERson, On the dynamics of strong temperature disturbances in the upper atmosphere of the Earth, Phys. Fluids A, 1 (1989), pp. 887-891.

[13] A. A. Samarski, V. A. Galaktionov, S. P. Kurdyumov, and A. P. Mikhailov, Blow- $U p$ in Quasilinear Parabolic Equations, Walter de Gruyter, New York, 1995.

[14] A. L. SÁnchez, A. Liñán, And F. A. Williams, Chain-branching explosions in mixing layers, SIAM J. Appl. Math., 59 (1999), pp. 1335-1355.

[15] C. SÁnchez-Tarifa, A. Crespo, and E. Fraga, A theoretical model for the combustion of droplets in supercritical conditions and gas pockets, Astronautica Acta, 17 (1972), pp. 685692.

[16] J. Sanz, A. Liñán, M. Rodríguez, And J. R. SAnmartín, Quasi-steady expansion of plasma ablated from laser-irradiated pellets, Phys. Fluids, 24 (1981), pp. 2098-2106.

[17] H. Schlichting, Boundary-Layer Theory, Springer-Verlag, Berlin, 2000. 
[18] L. I. Sedov, Propagation of strong shock waves, Prikl. Mat. Mekh., 10 (1946), pp. 241-250.

[19] G. I. TAYLOR, The formation of a blast wave by a very intense explosion. Part I. Theoretical discussion, Proc. Roy. Soc. London A, 201 (1950), pp. 159-174.

[20] G. I. TAYLOR, The formation of a blast wave by a very intense explosion. Part II. The atomic explosion of 1945, Proc. Roy. Soc. London A, 201 (1950), pp. 175-186.

[21] F. A. Williams, Combustion Theory, Benjamin Cummings, Menlo Park, CA, 1985.

[22] Ya. B. Zeldovich And A. S. Kompaneetz, Towards a theory of heat conduction with thermal conductivity depending on the temperature, Collection of papers dedicated to the 70th birthday of Academician A. F. Ioffe, Izd. Akad. Nauk SSSR, Moscow, 1950, pp. 61-71.

[23] Ya. B. Zeldovich and Yu. P. Raizer, Physics of Shock Waves and High Temperature Hydrodynamics Phenomena, Fizmatgiz, Moscow, 1963; English translation, Academic Press, New York, 1967. 